\title{
Likelihood theory for the graph Ornstein-Uhlenbeck process
}

\author{
Valentin Courgeau $^{1}$ (D) Almut E. D. Veraart ${ }^{1}$
}

Received: 7 July 2021 / Accepted: 21 September 2021 / Published online: 21 October 2021

(c) The Author(s) 2021

\begin{abstract}
We consider the problem of modelling restricted interactions between continuously-observed time series as given by a known static graph (or network) structure. For this purpose, we define a parametric multivariate Graph Ornstein-Uhlenbeck (GrOU) process driven by a general Lévy process to study the momentum and network effects amongst nodes, effects that quantify the impact of a node on itself and that of its neighbours, respectively. We derive the maximum likelihood estimators (MLEs) and their usual properties (existence, uniqueness and efficiency) along with their asymptotic normality and consistency. Additionally, an Adaptive Lasso approach, or a penalised likelihood scheme, infers both the graph structure along with the GrOU parameters concurrently and is shown to satisfy similar properties. Finally, we show that the asymptotic theory extends to the case when stochastic volatility modulation of the driving Lévy process is considered.
\end{abstract}

Keywords Ornstein-Uhlenbeck processes · Multivariate Lévy process · Continuous-time likelihood · Maximum likelihood estimator · Graphical modelling · Central limit theorem · Adaptive Lasso

\section{Introduction}

Ornstein-Uhlenbeck (OU) models, driven by Brownian motion or Lévy processes, form a class of continuous-time models with a broad range of applications: in finance for pairs trading (Endres and Stübinger 2019; Holý and Tomanová 2018) and volatility modelling (BarndorffNielsen and Shephard 2001; Pigorsch and Stelzer 2009b), in neuroscience (Melanson and Longtin 2019), or even in electricity management (Longoria et al. 2018). In parallel, highdimensional time series datasets fostered the development of sparse inference for OU-type processes (Boninsegna et al. 2018; Gaiffas and Matulewicz 2019) as a way to control interactions within complex systems. On the other hand, graphical time series models are usually restricted to discrete-time time series (Knight et al. 2016, 2020; Zhu et al. 2017).

From this observation, we introduce a mean-reverting process formulated as a graphical model and we derive its theoretical properties. This model contributes to the development of methodologies leveraging both the modelling flexibility of continuous-time models and the

Valentin Courgeau

valentin.courgeau15@imperial.ac.uk

1 Department of Mathematics, Imperial College London, 180 Queen's Gate, London SW7 2AZ, UK 
sparsity of graphical models. Furthermore, this article unlocks both hypothesis testing and uncertainty estimation in this setting.

In our framework, a graph is a set of nodes that interact together through a collection of links, or edges. Each node is described by a numerical value at any time and we observe a multivariate time series, where each component is the value of one node at a given time. To accommodate this graph structure, we introduce the Graph Ornstein-Uhlenbeck (GrOU) process, a parametric autoregressive model placed on a graph and driven by a Lévy process (Masuda 2004, 2007). This strictly stationary model quantifies the impact of a node value on its increments, the momentum effect, and the impact of the neighbouring nodes, the network effect. It is interpreted as a continuous-time extension to the discrete-time Network Vector Autoregression (NAR) model (Zhu et al. 2017) — a model used for the modelling of social media (Zhu et al. 2020), financial returns (Chen et al. 2020) or risk dynamics (Chen et al. 2019). We also introduce an alternative formulation with separate momentum and network effects for each node for a finer modelling of the graph dynamics.

For more details, the general multivariate Lévy-driven OU process and its ergodic properties are presented in Kevei (2018); Masuda (2004, 2007); Sandrić (2016). We refer to Sørensen (1991) for a general theory of the likelihood inference for continuously-observed jump diffusions.

This article focuses on providing the theoretical guarantees necessary for applications. First, we introduce the Lévy-driven GrOU model. Although the estimation of Lévy-driven OU processes have been largely considered (Gushchin et al. 2020; Masuda 2010), to the best of the authors' knowledge, the formulation of the GrOU process is the first of its kind.

Second, under non-degeneracy conditions, we show that this model belongs to an exponential family (Küchler and Sørensen 1997; Mancini 2009) with a fully-explicit likelihood function. We derive corresponding maximum likelihood estimators (MLEs) to estimate the momentum and networks effects. Those estimators are shown to be consistent and efficient in the sense of Hájek-Le Cam's convolution theorem (Hájek 1970; Le Cam and Lo Yang 1990). Those results extend the continuous-time asymptotic results given in the Lévy-driven univariate setting (Mai 2014) and the multivariate case with a Brownian noise (Basak and Lee 2008; Höpfner 2014).

Then, we prove that this family of models is locally asymptotically normal (LAN) (Le Cam and Lo Yang 1990) and provide a couple of central limit theorems (CLTs) under square integrability conditions. In addition, we extend the adaptive Lasso regularisation scheme from Gaïffas and Matulewicz (2019) to the Lévy-driven case and prove the asymptotic normality of the estimator. This approach can be used when the graph structure is not known a priori: our approach can not only be applied on graphical time series but also on general multivariate time series with sparse dependencies.

Finally, by assuming that the covariance matrix of the Lévy process is itself a matrixvalued OU process, i.e. both time-dependent and stochastic, the GrOU model is equipped with a stochastic covariance modulation term (Pigorsch and Stelzer 2009b) along with a pure-jump term. We show that the resulting process would still be ergodic using the mixing criterion from Fuchs and Stelzer (2013). The asymptotic results are shown to hold conditional on the knowledge of this volatility process and open the doors to a more complex modelling of graphical interactions of time series.

The GrOU process is presented in Sect. 2 where we describe formally the momentum and network effects along with non-degeneracy conditions. Then, Sect. 3 is devoted to setting up the likelihood framework and to proving the existence and uniqueness of the MLEs. In Sect. 4, both the LAN property of the model along with the Hájek-Le Cam efficiency of the MLEs are provided. We also present the MLE CLTs with an explicit limiting covariance matrix. We 
present an Adaptive Lasso scheme as well as extend known Brownian asymptotic properties to the Lévy-driven case in Sect. 5. Finally, in Sect. 6, we extend further our framework to include an OU-type stochastic volatility term and we show that, conditional on the knowledge of the stochastic volatility process, the central limit theorems from Sects. 4 and 5 hold.

\section{A graph Ornstein-Uhlenbeck process}

In this section, we define the Graph Ornstein-Uhlenbeck (GrOU) process to study either the aggregated or individual behaviour of nodes on the graph.

\subsection{Notations}

We consider a filtered probability space $\left(\Omega, \mathcal{F},\left(\mathcal{F}_{t}, t \in \mathbb{R}\right), \mathrm{P}_{0}\right)$ to which all stochastic processes are adapted. We consider two-sided Lévy processes $\left(\mathbb{L}_{t}, t \in \mathbb{R}\right)$ (i.e stochastic processes with stationary and independent increments and continuous in probability and $\mathbb{L}_{0}=\mathbf{0}_{\mathbf{D}}, \mathrm{P}_{0}-$ a.s.) (Brockwell 2009, Remark 1) which are without loss of generality assumed to be càdlàg and we write $\mathbb{Y}_{t-}:=\lim _{s \uparrow t} \mathbb{Y}_{s}$ for any $t \in \mathbb{R}$. For any probability measure $\mathrm{P}$, we denote by $\mathrm{P}_{t}$ its restriction to the $\sigma$-field $\mathcal{F}_{t}$ for any $t \in \mathbb{R}$.

We denote by det the matrix determinant, the space of $\{0,1\}$-valued $d \times d$ matrices by $\mathcal{M}_{d}(\{0,1\})$, the space of real-valued $d \times d$ (resp. $\left.n \times d\right)$ matrices by $\mathcal{M}_{d}(\mathbb{R})$ (resp. $\mathcal{M}_{n, d}(\mathbb{R})$ ), the linear subspace of $d \times d$ symmetric matrices by $\mathbb{S}_{d}$, the (closed in $\mathbb{S}_{d}$ ) positive semidefinite cone (i.e. with the real parts of their eigenvalues non-negative) by $\mathbb{S}_{d}^{+}$and the (open in $\mathbb{S}_{d}$ ) positive definite cone (i.e. with the real parts of their eigenvalues positive) by $\mathbb{S}_{d}^{++}$. In particular, $\mathbf{I}_{d \times d} \in \mathcal{M}_{d}(\mathbb{R})$ denotes the $d \times d$ identity matrix.

We denote by $\lambda^{l e b}$ the one-dimensional Lebesgue measure. For a non-empty topological space, $\mathcal{B}(S)$ is the Borel $\sigma$-algebra on $S$ and $\pi$ is some probability measure on $(S, \mathcal{B}(S))$. The collection of all Borel sets in $S \times \mathbb{R}$ with finite $\pi \otimes \lambda^{l e b}$-measure is written as $\mathcal{B}_{b}(S \times \mathbb{R})$. Also, the norms of vectors and matrices are denoted by $\|\cdot\|$. We usually take the Euclidean (or Frobenius) norm but due to the equivalence between norms, our results are not norm-specific and are valid under any norm in $\mathbb{R}^{d}$ or $\mathcal{M}_{d}(\mathbb{R})$.

In addition, for an invertible matrix $\boldsymbol{M} \in \mathcal{M}_{d}(\mathbb{R})$, we define $\langle\boldsymbol{x}, \boldsymbol{y}\rangle_{\boldsymbol{M}}:=\boldsymbol{x}^{\top} \boldsymbol{M}^{\mathbf{- 1}} \boldsymbol{y}$ for $\boldsymbol{x}, \boldsymbol{y} \in \mathbb{R}^{d}$. Finally, for a process $\mathbb{X}_{t}=\left(X_{t}^{(1)}, \ldots, X_{t}^{(d)}\right)^{\top} \in \mathbb{R}^{d}$ we denote by $[\mathbb{X}]_{t}$ the matrix $\left(\left[X^{(i)}, X^{(j)}\right]_{t}\right)$ of quadratic co-variations up to time $t \geq 0$.

In this article, $\otimes$ denotes the Kronecker matrix product, $\odot$ is for the Hadamard (elementwise) matrix product and vec is the vectorisation transformation where columns are stacked on one another. We denote the inverse vectorisation transformation by $\operatorname{vec}^{-1}(\boldsymbol{x}):=$ $\left(\operatorname{vec}\left(\mathbf{I}_{d \times d}\right)^{\top} \otimes \mathbf{I}_{d \times d}\right) \cdot\left(\mathbf{I}_{d \times d} \otimes \boldsymbol{x}\right) \in \mathcal{M}_{d}(\mathbb{R})$ for $\boldsymbol{x} \in \mathbb{R}^{d^{2}}$.

\subsection{The Lévy-driven Ornstein-Uhlenbeck process}

We recall the construction of Ornstein-Uhlenbeck (OU) processes and introduce their graphical interpretation, namely the GrOU process. We give two parametrisations specific to the modelling of graph structures namely the $\boldsymbol{\theta}$-GrOU and the $\boldsymbol{\psi}$-GrOU: the former encodes the momentum and network effects for the whole graph into two scalar parameters while the latter has separate parameters for each node and each neighbour. 
We consider a $d$-dimensional OU process $\mathbb{Y}_{t}=\left(Y_{t}^{(1)}, \ldots, Y_{t}^{(d)}\right)^{\top}$ for $t \geq 0$ satisfying the stochastic differential equation (SDE) for a dynamics matrix $\mathbf{Q} \in \mathbb{S}_{d}^{++}$

$$
d \mathbb{Y}_{t}=-\mathbf{Q} \mathbb{Y}_{t-} d t+d \mathbb{L}_{t},
$$

for a two-sided $d$-dimensional Lévy process $\mathbb{L}_{t}=\left(L_{t}^{(1)}, \ldots, L_{t}^{(d)}\right)^{\top}$ (Brockwell 2009, Remark 1) such that $\mathbb{Y}_{0}$ is independent of $\left(\mathbb{L}_{t}, t \geq 0\right.$ ) (Masuda 2004, Section 1).

The Lévy process $\mathbb{L}$ is defined by the Lévy-Khintchine characteristic triplet $(\boldsymbol{b}, \boldsymbol{\Sigma}, v)$ with respect to the truncation function $\tau(z):=\mathbb{I}_{\left\{\boldsymbol{x} \in \mathbb{R}^{d}:\|\boldsymbol{x}\| \leq 1\right\}}(\boldsymbol{z})$ where $\mathbb{I}$ denotes the indicator function. More explicitly, the Lévy-Khintchine representation yields, for $t \in \mathbb{R}$,

$$
\begin{aligned}
& \mathrm{E}\left[\exp \left(i \boldsymbol{u}^{\top} \mathbb{L}_{t}\right)\right] \\
& \quad=\exp \left\{t\left(i \boldsymbol{u}^{\top} \boldsymbol{b}-\frac{1}{2} \boldsymbol{u}^{\top} \boldsymbol{\Sigma} \boldsymbol{u}+\int_{\mathbb{R}^{d} \backslash\left\{\boldsymbol{0}_{d}\right\}}\left[\exp \left(i \boldsymbol{u}^{\top} \boldsymbol{z}\right)-1-i \boldsymbol{u}^{\top} \boldsymbol{z} \tau(\boldsymbol{z})\right] d v(\boldsymbol{z})\right)\right\},
\end{aligned}
$$

where $\boldsymbol{u}, \boldsymbol{b} \in \mathbb{R}^{d}, \boldsymbol{\Sigma} \in \mathbb{S}_{d}^{++}$and $v$ is a Lévy measure on $\mathbb{R}^{d}$ satisfying $\int_{\mathbb{R}^{d} \backslash\{\boldsymbol{0}\}}(1 \wedge$ $\left.\|z\|^{2}\right) v(d z)<\infty$.

Again, without loss of generality, consider that $\mathrm{P}_{0}$ is a probability measure where $\left(\mathbb{L}_{t}, t \in\right.$ $\mathbb{R}$ ) is a càdlàg Lévy process as mentioned above. Also, we denote by $\mathrm{P}_{t, 0}$ the probability measure $P_{0}$ restricted to the $\sigma$-field $\mathcal{F}_{t}$ as introduced in Sect. 2.1.

\subsection{The OU process on a graph}

The components of $\mathbb{Y}$ are interpreted as the nodes of a graph structure linked together through a collection of edges. Those are given in a adjacency (or graph topology) matrix $\mathbf{A}=\left(a_{i j}\right) \in$ $\mathcal{M}_{d}(\{0,1\}):: a_{i j}=1$ for an existing link between node $i$ and $j, 0$ otherwise. We assume that $a_{i i}=0$ for all $i \in\{1, \ldots, d\}$. Requiring the knowledge of the adjacency matrix is necessary for the graphical interpretation of the OU process. This limitation is alleviated in the sparse inference scheme introduced in Sect. 5.

Assumption 1 We assume that $\mathbf{A}$ is deterministic, static in time and known.

For $i \in\{1, \ldots, d\}$, define $n_{i}:=1 \vee \sum_{j \neq i} a_{i j}$, which counts the number of neighbouring nodes the $i$-th node is connected to, or node degree. We can now define the row-normalised adjacency matrix

$$
\overline{\mathbf{A}}:=\operatorname{diag}\left(n_{1}^{-1}, \ldots, n_{d}^{-1}\right) \mathbf{A} .
$$

to normalise the parameter values representing the momentum and networks effects which we introduce below.

The $\boldsymbol{\theta}$-GrOU process We introduce the two-dimensional parameter vector $\boldsymbol{\theta}:=\left(\theta_{1}, \theta_{2}\right)^{\top} \in$ $\mathbb{R}^{2}$ describing the network effect and the momentum effect respectively, and define the matrix

$$
\mathbf{Q}=\mathbf{Q}(\boldsymbol{\theta}):=\theta_{2} \mathbf{I}_{d \times d}+\theta_{1} \overline{\mathbf{A}}
$$

The row-normalised adjacency matrix $\overline{\mathbf{A}}$ makes the momentum parameter $\theta_{2}$ and the network parameter $\theta_{1}$ in (2) directly comparable with one another independently of the node degrees. This yields the SDE given by

$$
d \mathbb{Y}_{t}=-\mathbf{Q}(\boldsymbol{\theta}) \mathbb{Y}_{t-} d t+d \mathbb{L}_{t},
$$


whose $i$-th component satisfies the equation

$$
d Y_{t}^{(i)}=-\theta_{2} Y_{t}^{(i)} d t-\theta_{1} n_{i}^{-1} \sum_{j \neq i} a_{i j} Y_{t}^{(j)} d t+d L_{t}^{(i)}, t \geq 0 .
$$

The $\psi$-GrOU process Recall that we denote by $\odot$ the Hadamard product and by $\mathrm{vec}^{-1}$ the inverse vectorisation transformation. In general, for a $d^{2}$-dimensional vector $\psi \in \mathbb{R}^{d^{2}}$, we have

$$
\mathbf{Q}=\mathbf{Q}(\boldsymbol{\psi}):=\left(\mathbf{I}_{d \times d}+\overline{\mathbf{A}}\right) \odot \operatorname{vec}^{-1}(\boldsymbol{\psi}),
$$

where

$$
\operatorname{vec}^{-1}(\boldsymbol{\psi})=\left(\begin{array}{ccc}
\psi_{1} & \ldots & \psi_{d(d-1)+1} \\
\vdots & \ddots & \vdots \\
\psi_{d} & \ldots & \psi_{d \times d}
\end{array}\right),
$$

such that the corresponding SDE is written as:

$$
d Y_{t}^{(i)}=-Q_{i i} Y_{t}^{(i)} d t-\sum_{j \neq i} Q_{i j} Y_{t}^{(j)} d t+d L_{t}^{(i)}, \quad t \geq 0 .
$$

Formally, we obtain

$$
d Y_{t}^{(i)}=-\underbrace{\boldsymbol{\psi}_{d(i-1)+i}}_{\text {momentum effect }} Y_{t}^{(i)} d t-\underbrace{-n_{i}^{-1} \sum_{j \neq i} a_{i j} \boldsymbol{\psi}_{d(j-1)+i}}_{\text {network effect }} Y_{t}^{(j)} d t+d L_{t}^{(i)}, \quad t \geq 0 .
$$

This second parametrisation alleviates the scarcity of network interactions imposed by $\boldsymbol{\theta}$ yet exposes the estimation to the curse of dimensionality as the number of nodes $d$ grows. For simplicity, one may write $\mathbf{Q}$ for $\mathbf{Q}(\boldsymbol{\theta})$ or $\mathbf{Q}(\boldsymbol{\psi})$ when the context is clear.

With $\mathbf{Q}(\boldsymbol{\theta})$, we restrict the interactions to the network and momentum effects. This extends the current framework to partially observable networks (e.g. too large for computations) where an exploration process needs to take place (Dereich and Mörters 2013, Section 5). This makes the estimation robust again the curse of dimensionality coming from the number of nodes. We now define the GrOU process as follows.

Definition 1 The Graph Ornstein-Uhlenbeck (GrOU) process is a càdlàg process $\left(\mathbb{Y}_{t}, t \geq 0\right)$ satisfying Eq. (1) for some two-sided Lévy noise $\left(\mathbb{L}_{t}, t \in \mathbb{R}\right)$ where $\mathbf{Q}$ is given by either Eq. (2) or by Eq. (3) such that $\mathbf{Q}$ is positive definite. This process is then called a $\boldsymbol{\theta}$-GrOU process or a $\psi$-GrOU process, respectively.

We give sufficient conditions for $\mathbf{Q}$ to be positive definite in both cases in Sect. 2.4.

\subsection{Stationary solution}

Recall that $\mathbb{S}_{d}^{++}$is the set of $d \times d$ matrices such that the real parts of the eigenvalues are positive. We restrict ourselves to study strictly stationary time series, we give the known conditions under which the OU process is strictly stationary.

Remark 2.4.1 Note that $\mathbf{Q} \in \mathbb{S}_{d}^{++}$if and only if $\operatorname{det}\left(e^{-t \mathbf{Q}}\right) \stackrel{t \rightarrow+\infty}{\longrightarrow} 0$ (Masuda 2004).

Given the standard OU processes theory from Brockwell et al. (2007), Masuda (2004), we assume the following: 
Assumption 2 Suppose that $\mathbf{Q} \in \mathbb{S}_{d}^{++}$and that the Lévy measure $v(\cdot)$ satisfies the log moment condition:

$$
\int_{\|z\|>1} \ln \|z\| v(d z)<\infty
$$

Then, under Assumption 2, there is a unique strictly stationary solution of the above SDE given by

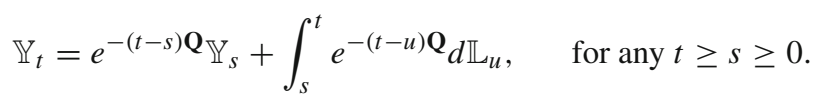

Recall that the Lévy-Khintchine characteristic triplet of $\mathbb{L}$ with respect to the truncation function $\tau$ is denoted $(\boldsymbol{b}, \boldsymbol{\Sigma}, v)$. Proposition 2.1, Masuda (2004) yields that the transition probability from $\boldsymbol{x}$ at time $t$ denoted $\mathrm{P}(t, \boldsymbol{x}, \cdot)$ is characterised by the triplet $\left(b_{t, \boldsymbol{x}}, C_{t}, v_{t}\right)$ (with respect to $\tau$ ) defined as

$$
\begin{aligned}
& b_{t, \boldsymbol{x}}:=e^{-t \mathbf{Q}} x+\int_{0}^{t} e^{-s \mathbf{Q}_{b}} d s+\int_{\mathbb{R}^{d}} \int_{0}^{t} e^{-s \mathbf{Q}} z\left[\tau\left(e^{-s \mathbf{Q}} z\right)-\tau(z)\right] d s v(d z), \\
& C_{t}:=\int_{0}^{t} e^{-s \mathbf{Q}} C e^{-s \mathbf{Q}^{\top}} d s, \quad v_{t}(S):=\int_{0}^{t} v\left(e^{s \mathbf{Q}} S\right) d s, \quad \text { for any } S \in \mathcal{B}\left(\mathbb{R}^{d}\right),
\end{aligned}
$$

where the limit as $t \rightarrow \infty$ leads a characteristic triplet of the form $\left(\boldsymbol{b}_{\infty}, C_{\infty}, v_{\infty}\right)$ which characterises the unique invariant distribution of $\mathbb{Y}$ denoted by $\pi$ i.e. $\mathbb{Y}_{t} \stackrel{\mathcal{D}}{\longrightarrow} \mathbb{Y}_{\infty} \sim \pi$ as $t \rightarrow \infty$.

Proposition 2.4.2 If $\theta_{2}>0$ such that $\theta_{2}>\left|\theta_{1}\right|$, then $\boldsymbol{Q}(\boldsymbol{\theta}) \in \mathbb{S}_{d}^{++}$.

Proof Recall that the Geršgorin's circle theorem states that any eigenvalue of $\mathbf{Q}(\boldsymbol{\theta})$ is found in a closed circle of centre $Q_{i i}=\theta_{2}$ and radius equal to the sum of non-diagonal entries of the $i$-th row $\sum_{j \neq i}\left|Q_{i j}\right|$ for some $i \in\{1, \ldots, d\}$. Since $\overline{\mathbf{A}}$ is row-normalised and given that $\theta_{2}>\left|\theta_{1}\right|$, we conclude that the eigenvalues must be in an open disk with center $\theta_{2}$ and radius $\left|\theta_{1}\right|$. This disk is positioned in the positive half-plane and does not contain the origin. This yields that all eigenvalues are strictly positive.

Proposition 2.4.2 makes practical sense as it requires that the auto-regressive part of the model is predominant in absolute terms. For the $\boldsymbol{\psi}$-GrOU formulation, we obtain the following:

Proposition 2.4.3 If we have

$$
\psi_{i(d-1)+i}>0 \text { and } \psi_{d(i-1)+i}>n_{i}^{-1} \sum_{j \neq i}\left|\psi_{d(j-1)+i}\right| \text { for any } i \in\{1, \ldots, d\},
$$

then $\boldsymbol{Q}(\boldsymbol{\psi}) \in \mathbb{S}_{d}^{++}$. This means that $\operatorname{vec}^{-1}(\boldsymbol{\psi}) \in \mathcal{M}_{d}(\mathbb{R})$ has positive diagonal elements.

Proof The absolute sum of the off-diagonal elements of the $i$-th row of $\mathbf{Q}(\boldsymbol{\psi})$ give

$$
\sum_{j \neq i}\left|Q_{i j}\right|=n_{i}^{-1} \sum_{j \neq i} a_{i j}\left|\psi_{d(j-1)+i}\right| \leq n_{i}^{-1} \sum_{j \neq i}\left|\psi_{d(j-1)+i}\right|<\left|\psi_{d(i-1)+i}\right| .
$$

Similarly to the proof of Proposition 2.4.2, applying Geršgorin's circle theorem yields the expected result. 
Remark 2.4.4 The model defined in (1) is a generalisation of the (discrete-time) Vector Autoregressive (VAR) model (Sims 1980) with a depth of 1 . For a step size $\Delta>0$, we then denote the sampled process by $\mathbb{X}_{j}:=\left(X_{j}^{(1)}, \ldots, X_{j}^{(d)}\right)^{\top}$ for $j \in \mathbb{N}$, where $X_{j}^{(k)}=Y_{j \Delta}^{(k)}$. Hence we have the VAR(1)-representation

$$
\mathbb{X}_{j}=\boldsymbol{\Phi} \mathbb{X}_{j-1}+\mathbb{Z}_{j}, \quad j \in \mathbb{N},
$$

where the parameter matrix $\boldsymbol{\Phi}$ is given by

$$
\boldsymbol{\Phi}=e^{-\Delta \mathbf{Q}}=e^{-\Delta\left(\theta_{1} \overline{\mathbf{A}}+\theta_{2} \mathbf{I}_{d \times d}\right)}=e^{-\Delta \theta_{1} \overline{\mathbf{A}}} e^{-\Delta \theta_{2} \mathbf{I}_{d \times d}},
$$

and an i.i.d. noise sequence given by

$$
\mathbb{Z}_{j}=\int_{(j-1) \Delta}^{j \Delta} e^{-(j \Delta-u) \mathbf{Q}} d \mathbb{L}_{u} .
$$

This VAR-like formulation with a fixed step size $\Delta$ is studied in Fasen (2013) where one estimates $e^{-\Delta \mathbf{Q}}$ as a $d \times d$ matrix directly. However, a strong identifiability issue hinders the estimation of $\mathbf{Q}$ from $e^{-\Delta \mathbf{Q}}$ : the logarithm of a matrix (or log-matrix) is not necessarily itself a real matrix and, if so, it may be not unique (Culver 1966, p. 1146). The former holds if and only if $e^{-\Delta \mathbf{Q}}$ is nonsingular and each elementary divisor (Jordan block) of $e^{-\Delta \mathbf{Q}}$ belonging to a negative eigenvalue occurs an even number of times (Culver 1966, Th. 1). Since $e^{-\Delta \mathbf{Q}}$ has only positive eigenvalues, a real-valued log-matrix always exist. On the other hand, the uniqueness is more difficult to ascertain. It requires that all the eigenvalues of $e^{-\Delta \mathbf{Q}}$ are positive and real and that no elementary divisor (or Jordan block) of $e^{-\Delta \mathbf{Q}}$ belonging to any eigenvalue appears more than once (Culver 1966, Th. 2). Those conditions are difficult to check in practice: the eigenvalues $\lambda$ of $\mathbf{Q}$ have positive real parts but may have non-zero imaginary parts such that $e^{-\Delta \lambda}$ may be non-real. Also, the Jordan block assumption does not hold in general for all $\mathbf{Q} \in \mathbb{S}_{d}^{++}$. With this issue in mind, we introduce a fully-explicit likelihood function in Sect. 3; whose logarithm is well-defined as a real-valued positive function.

\section{Likelihood and estimators}

Suppose we observe the process $\mathbb{Y}$ continuously on $[0, T]$ for $T \in \mathbb{R} \cup\{\infty\}$ and let $t \in$ $[0, T]$. In this section, we present the likelihood framework of interest and derive closedform formulas for the $\boldsymbol{\theta}$-GrOU and $\boldsymbol{\psi}$-GrOU MLEs.

\subsection{Ergodicity}

Recall the ergodic theorem of a process $\left(\mathbb{Y}_{t}, t \in \mathbb{R}\right)$ satisfying Eq. (1) is given by:

Proposition 3.1.1 (Theorems 2.1 and 2.6, Masuda (2007)) Suppose that Assumptions 1 and 2 hold. Then $\left(\mathbb{Y}_{t}, t \in \mathbb{R}\right)$ admits a unique invariant distribution $\pi$ for any choice of the law $\eta$ of the initial value $\mathbb{Y}_{0}$. Moreover, for any measurable function $g: \mathbb{R}^{d} \mapsto \mathbb{R}^{k}$ satisfying $\mathbb{E}\left[\left\|g\left(\mathbb{Y}_{\infty}\right)\right\|\right]:=\int_{\mathbb{R}^{d}}\|g(y)\| \pi(d y)<\infty$ and for any $\eta$, we have

$$
\frac{1}{t} \int_{0}^{t} g\left(\mathbb{Y}_{s}\right) d s \stackrel{t \rightarrow \infty}{\longrightarrow} \mathbb{E}\left[g\left(\mathbb{Y}_{\infty}\right)\right]:=\int_{\mathbb{R}^{d}} g(y) \pi(d y), \quad P_{\eta}-\text { a.s. }
$$

where $P_{\eta}$ is the law of $\mathbb{Y}$ associated with the initial value $\mathbb{Y}_{0} \sim \eta$. 
According to Sect. 2.4, recall that $\mathbb{Y}_{t} \stackrel{\mathcal{D}}{\longrightarrow} \mathbb{Y}_{\infty} \sim \pi$ as $t \rightarrow \infty$ where $\pi$ is the invariant distribution characterised by $\left(\boldsymbol{b}_{\infty}, C_{\infty}, v_{\infty}\right)$. This result is essential for the asymptotic properties of the model statistical inference and was further extended in Kevei (2018); Sandrić (2016). We present the general likelihood framework used for GrOU processes.

\subsection{The fully-explicit likelihood function}

To infer the model parameters, we set up an explicit likelihood function to be maximised. For a general positive definite dynamics matrix $\mathbf{Q}$, the Radon-Nikodym derivative of the corresponding $d$-dimensional Ornstein-Uhlenbeck process (Eq. 1) is expressed as follows (Mai 2014; Pap and van Zuijlen 1996):

$$
\frac{d P_{t, \mathbb{Y}}}{d \mathrm{P}_{t, 0}}=\exp \left\{-\int_{0}^{t}\left\langle\mathbf{Q} \mathbb{Y}_{s}, d \mathbb{Y}_{s}^{c}\right\rangle_{\Sigma}-\frac{1}{2} \int_{0}^{t}\left\langle\mathbf{Q} \mathbb{Y}_{s}, \mathbf{Q} \mathbb{Y}_{s}\right\rangle_{\Sigma} d s\right\}, \quad t \in[0, T],
$$

where $\mathbb{Y}_{s}^{c}$ is the continuous $\mathrm{P}_{0}$-martingale part and $\mathrm{P}_{\mathbb{Y}}$ is a probability measure equivalent to $\mathrm{P}_{0}$ such that the process in Eq. (6) is a martingale. Similarly to $\mathrm{P}_{t, 0}, \mathrm{P}_{t, \mathbb{Y}}$ is the restriction of $\mathrm{P}_{\mathbb{Y}}$ on $\mathcal{F}_{t}$ for any $t \in \mathbb{R}$. We note that, since $\boldsymbol{\Sigma} \in \mathbb{S}_{d}^{++}, \boldsymbol{\Sigma}$ is invertible and hence Eq. (6) is well-defined.

Remark 3.2.1 In the rest of the article, we write $t=T$ (i.e. $t$ is the time horizon itself) and consider the likelihood at time $t$ directly.

\subsection{The case of the $\theta$-GrOU process}

In this section, we write the likelihood for $\boldsymbol{\theta}$-GrOU processes and obtain the corresponding maximum likelihood estimator along with its existence and uniqueness. Consider the notations:

Notation 3.3.1 Define the deterministic positive definite matrix

$$
\boldsymbol{G}_{\infty}:=\left(\begin{array}{cc}
E\left(\left\langle\overline{\mathbf{A}} \mathbb{Y}_{\infty}, \overline{\mathbf{A}} \mathbb{Y}_{\infty}\right\rangle_{\boldsymbol{\Sigma}}\right) & E\left(\left\langle\overline{\mathbf{A}} \mathbb{Y}_{\infty}, \mathbb{Y}_{\infty}\right\rangle_{\boldsymbol{\Sigma}}\right) \\
E\left(\left\langle\overline{\mathbf{A}} \mathbb{Y}_{\infty}, \mathbb{Y}_{\infty}\right\rangle_{\boldsymbol{\Sigma}}\right) & E\left(\left\langle\mathbb{Y}_{\infty}, \mathbb{Y}_{\infty}\right\rangle_{\boldsymbol{\Sigma}}\right)
\end{array}\right)
$$

and define

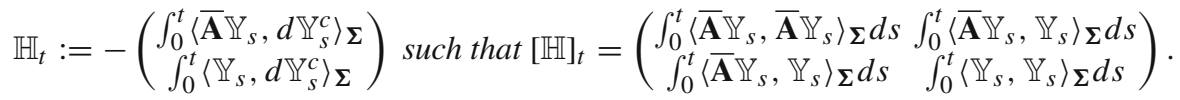

Using Eq. (2), we deduce that

$$
\int_{0}^{t}\left\langle\mathbf{Q}(\boldsymbol{\theta}) \mathbb{Y}_{s}, \mathbf{Q}(\boldsymbol{\theta}) \mathbb{Y}_{s}\right\rangle_{\Sigma} d s=\boldsymbol{\theta}^{\top} \cdot[\mathbb{H}]_{t} \cdot \boldsymbol{\theta} .
$$

Lemma 3.3.2 Suppose that Assumptions 1 and 2 hold and that $\mathbb{Y}$ has finite second moments. Then, we have that $t^{-1}[\mathbb{H}]_{t}$ converges almost surely to $\boldsymbol{G}_{\infty}$ as $t \rightarrow \infty$. Therefore, $[\mathbb{H}]_{t}=$ $O(t)$ componentwise as $t \rightarrow \infty$ and we obtain that $E\left(\left|[\mathbb{H}]_{t}\right|\right) \rightarrow \infty$ componentwise as $t \rightarrow \infty$.

Proof By stationarity and ergodicity, $t^{-1}[\mathbb{H}]_{t}$ converges almost surely to $\boldsymbol{G}_{\infty}$ as $t \rightarrow \infty$. This matrix has finite elements since $\mathbb{Y}$ has finite second moments. By Jensen's inequality, we obtain $\mathrm{E}\left(\left|\mathbb{H}_{t}\right|\right) \geq\left|\mathrm{E}\left([\mathbb{H}]_{t}\right)\right|$. By Fubini's theorem and stationarity we have that $\mathrm{E}\left([\mathbb{H}]_{t}\right)=$ $\int_{0}^{t} \boldsymbol{G}_{\infty} d t=O(t)$ componentwise as $t \rightarrow \infty$ and hence $\mathrm{E}\left(\left|[\mathbb{H}]_{t}\right|\right) \rightarrow \infty$ componentwise as $t \rightarrow \infty$. 
We then set up a likelihood framework (in the sense of Section 2, Morales et al. (2000)) for the $\boldsymbol{\theta}$-GrOU process as follows:

Proposition 3.3.3 From Eq. (6), we obtain the likelihood ratio $\mathcal{L}_{t}(\boldsymbol{\theta} ; \mathbb{Y})$ :

$$
\mathcal{L}_{t}(\boldsymbol{\theta} ; \mathbb{Y}):=\frac{d P_{t, \mathbb{Y}}}{d P_{t, 0}}=\exp \left(\boldsymbol{\theta}^{\top} \mathbb{H}_{t}-\frac{1}{2} \boldsymbol{\theta}^{\top} \cdot[\mathbb{H}]_{t} \cdot \boldsymbol{\theta}\right), \quad \boldsymbol{\theta} \in \mathbb{R}^{2} .
$$

Additionally, $t \mapsto \mathbb{H}_{t}$ and $t \mapsto \boldsymbol{\theta}^{\top} \cdot[\mathbb{H}]_{t} \cdot \boldsymbol{\theta}$ (for a fixed $\boldsymbol{\theta}$ ) are càdlàg hence bounded and $\mathcal{F}_{t}$-measurable for any finite $t \in R$.

Proof According to Lemma 3.3.2 and by continuity, $\mathbb{H}$ and $[\mathbb{H}]$ are finite for any $t \geq t_{0}$ where $t_{0}$ is such that both $\mathbb{H}_{t_{0}}$ and $[\mathbb{H}]_{t_{0}}$ are finite. Also, $t \mapsto[\mathbb{H}]_{t}$ has a linear growth as $t \rightarrow \infty$. Therefore, the likelihood is defined on $\boldsymbol{\Theta}:=\left\{\boldsymbol{\theta} \in \mathbb{R}^{2}:\left|\boldsymbol{\theta}^{\top} \cdot[\mathbb{H}]_{t} \cdot \boldsymbol{\theta}\right|<\infty, \forall t \geq 0\right\}=\mathbb{R}^{2}$. By the properties of the stochastic integral with respect to the continuous martingale part of $\mathbb{Y}, t \mapsto \mathbb{H}_{t}$ is indeed càdlàg.

From Proposition 2.4.2, we define a compact set $\widehat{\boldsymbol{\Theta}}$ such that

$$
\widehat{\boldsymbol{\Theta}} \subseteq\left\{\left(\theta_{1}, \theta_{2}\right)^{\top} \in \mathbb{R}^{2}: \theta_{2}>0 \text { and } \theta_{2}>\left|\theta_{1}\right|\right\} .
$$

We state the main result on the existence and uniqueness of the continuous-time Maximum Likelihood Estimator (MLE) using Notation 3.3.1:

Theorem 3.3.4 ( $\boldsymbol{\theta}$-GrOU MLE with continuous-time observations) Suppose that Assumptions 1 and 2 hold. Assume that the $\boldsymbol{\theta}$-GrOU process $\mathbb{Y}$ is observed in continuous time and has finite second moments. Then, the MLE $\widehat{\boldsymbol{\theta}}_{t}$ on the compact set $\widehat{\boldsymbol{\Theta}}$ solves the equation

$$
\mathbb{H}_{t}=[\mathbb{H}]_{t} \cdot \widehat{\boldsymbol{\theta}}_{t}, \quad \text { for } t \geq 0 \text {. }
$$

Moreover, $\widehat{\boldsymbol{\theta}}_{t}$ satisfies the properties:

1. We have $\operatorname{det}\left([\mathbb{H}]_{t}\right)>0 P_{t, \mathbb{Y}}-$ a.s. for $t \geq 0$ large enough and

$$
\widehat{\boldsymbol{\theta}}_{t}=[\mathbb{H}]_{t}^{-1} \cdot \mathbb{H}_{t} \text {. }
$$

2. The MLE $\widehat{\boldsymbol{\theta}}_{t}$ exists almost surely and uniquely under $P_{t, \mathbb{Y}}$.

Proof See Appendix A.2.

This concludes the presentation of the two-parameter estimator and the MLE for the $\psi$-GrOU process is defined next.

\subsection{The case of the $\Psi$-GrOU process}

We focus on the $\psi$-GrOU processes and define their likelihood along with the corresponding MLE. From Basak and Lee (2008), one deduces an intermediary result:

Lemma 3.4.1 (Adapted from Theorem 4.1, Basak and Lee (2008)) Consider the $d \times d$ matrix $\mathbf{K}_{t}:=\int_{0}^{t} \mathbb{Y}_{s} \mathbb{Y}_{s}^{\top} d s$. This matrix is $P_{0}$-almost surely nonsingular for $t$ large enough in the sense that $\liminf _{t \rightarrow \infty} t^{-1} \lambda_{\min }\left(\mathbf{K}_{t}\right)>0$ and we also have that $\lambda_{\text {max }}\left(\mathbf{K}_{t}\right)=O(t) P_{0}-$ a.s. Here, $\lambda_{\min }$ and $\lambda_{\max }$ are respectively the smallest and largest eigenvalues (which are real since $\mathbf{K}_{t}$ is symmetric).

Proof See Appendix A.3. 
We define the equivalent matrix to $\mathbb{H}_{t}$ as follows

Definition 2 We define the node-level integrated response vector as $\mathbb{I}_{t}:=-\int_{0}^{t} \mathbb{Y}_{s} \otimes \Sigma^{-1} d \mathbb{Y}_{s}^{c}$ such that $[\mathbb{I}]_{t}:=\mathbf{K}_{t} \otimes \boldsymbol{\Sigma}^{-1}$ for any $t \geq 0$.

As hinted by Eq. (3), we first derive the likelihood under unrestricted network interactions before applying the network topology (as a linear transformation of the former). The corresponding $\psi$-GrOU likelihood is formulated as follows:

Proposition 3.4.2 We consider the dynamics $d \mathbb{Y}_{t}=-\mathrm{vec}^{-1}(\boldsymbol{\psi}) \cdot \mathbb{Y}_{t-} d t+d \mathbb{L}_{t}$ for some general parameter $\psi \in \mathbb{R}^{d^{2}}$ such that Proposition 2.4.3 holds. The likelihood with respect to $\psi$ is given by

$$
\mathcal{L}_{t}(\boldsymbol{\psi} ; \mathbb{Y})=\exp \left(\boldsymbol{\psi}^{\top} \cdot \mathbb{I}_{t}-\frac{1}{2} \boldsymbol{\psi}^{\top} \cdot[\mathbb{I}]_{t} \cdot \boldsymbol{\psi}\right), \quad \boldsymbol{\psi} \in \mathbb{R}^{d^{2}} .
$$

Proof See Appendix A.4.

Remark 3.4.3 To obtain the MLE of $\operatorname{vec}(\mathbf{Q}(\psi))$, we factor in the network topology $\overline{\mathbf{A}}$ by transforming linearly $\boldsymbol{\psi}$ into $\operatorname{vec}\left(\mathbf{I}_{d \times d}+\overline{\mathbf{A}}\right) \odot \boldsymbol{\psi}$ as given in Eq. (3). Therefore, there is no need to reformulate the likelihood given in Proposition 3.4.2.

Define a compact set $\widehat{\Psi} \subseteq \mathbb{R}^{d^{2}}$ such that

$$
\widehat{\boldsymbol{\Psi}} \subseteq\left\{\psi \in \mathbb{R}^{d^{2}}: \psi_{d(i-1)+i}>n_{i} \sum_{j \neq i}\left|\psi_{d(j-1)+i}\right|>0, \forall i \in\{1, \ldots, d\}\right\},
$$

that is where $\mathbf{Q}(\boldsymbol{\psi})$ is diagonally dominant: this ensures the well-definedness of the GrOU process. Similarly to the $\boldsymbol{\theta}$-GrOU case, we denote by $\left(\mathrm{P}_{\mathbb{Y}}^{\psi}, \boldsymbol{\psi} \in \widehat{\boldsymbol{\Psi}}\right)$ the statistical models of Lévy-driven Ornstein-Uhlenbeck processes with respect to the likelihood from Proposition 3.4.2 indexed on $\widehat{\Psi}$.

We formulate an equivalent to Theorem 3.3.4 for the $\psi$-GrOU process:

Theorem 3.4.4 ( $\psi$-GrOU MLE with continuous-time observations) Suppose that Assumptions 1 and 2 hold. Assume that the $\psi$-GrOU process $\mathbb{Y}$ is observed in continuous time and has finite second moments. Then, the MLE $\widehat{\boldsymbol{\psi}}_{t}$ on the compact set $\widehat{\boldsymbol{\Psi}}$ solves the equation

$$
\mathbb{I}_{t}=[\mathbb{I}]_{t} \cdot \widehat{\psi}_{t}, \quad \text { for } t \geq 0 \text {. }
$$

Moreover, $\widehat{\psi}_{t}$ satisfies the properties:

1. Since $\operatorname{det}\left([\mathbb{I}]_{t}\right)>0 P_{t, \mathbb{Y}}-$ a.s. for $t \geq 0$ large enough and

$$
\widehat{\boldsymbol{\psi}}_{t}=[\mathbb{I}]_{t}^{-1} \cdot \mathbb{I}_{t} .
$$

2. The MLE $\widehat{\boldsymbol{\psi}}_{t}$ exists almost surely and uniquely under $P_{t, \mathbb{Y}}$.

Proof Recall that $\int_{0}^{t} \mathbb{Y}_{s} \mathbb{Y}_{s}^{\top} d s$ is $\mathrm{P}_{t, \mathbb{Y}}-$ a.s. invertible (see Lemma 3.4.1). The same argument as for Theorem 3.3.4 can be applied. 


\section{Asymptotic theory for the MLEs}

Asymptotic properties of MLEs are a necessary step into formulating hypothesis tests necessary for sound inference. In this section, we consider having access to a continuous flow of data and we derive the asymptotic normality of the afore-mentioned estimators as well as an augmented estimator on the whole $\mathbf{Q}$ matrix. We consider the estimators efficiency in the sense of Hájek-Le Cam's convolution theorem (Hájek 1970, Section 2) under local asymptotic normality (Le Cam and Lo Yang 1990, Chapter 5, Section 6).

\subsection{Asymptotics for $\theta$-GrOU}

We now prove the consistency of the MLE for the $\boldsymbol{\theta}$-GrOU process on a compact set $\widehat{\boldsymbol{\Theta}}$.

Proposition 4.1.1 (Consistency of the estimator) Suppose Assumptions 1 and 2 hold. Suppose that $\left(\mathbb{Y}_{t}, t \geq 0\right.$ ) satisfies Eq. (1) and has finite second moments for $\boldsymbol{\theta} \in \widetilde{\boldsymbol{\Theta}}$ where $\widetilde{\boldsymbol{\Theta}}$ is a compact set in $\boldsymbol{\Theta}$. The MLE $\widehat{\boldsymbol{\theta}}_{t}$ is a consistent estimator under $P_{\mathbb{Y}}$ in the sense that $\widehat{\boldsymbol{\theta}}_{t} \stackrel{p}{\longrightarrow} \boldsymbol{\theta}$ as $t \rightarrow \infty$.

Proof See Appendix A.5.

We denote by $\left(\mathrm{P}_{\mathbb{Y}}^{\boldsymbol{\theta}}, \boldsymbol{\theta} \in \widehat{\boldsymbol{\Theta}}\right)$ the statistical models of Lévy-driven Ornstein-Uhlenbeck processes with respect to the likelihood from Proposition 3.3.3 indexed on $\widehat{\boldsymbol{\Theta}}$. We obtain the local asymptotic normality for this sequence:

Lemma 4.1.2 The family of statisticalmodels $\left(P_{\mathbb{Y}}^{\boldsymbol{\theta}}, \boldsymbol{\theta} \in \widehat{\boldsymbol{\Theta}}\right)$ with respect to $\left(\mathcal{L}_{t}(\boldsymbol{\theta} ; \mathbb{Y}): \boldsymbol{\theta} \in \widehat{\boldsymbol{\Theta}}\right)$ is locally asymptotically normal.

Proof See Appendix A.6

The central limit theorem for $\widehat{\boldsymbol{\theta}}$ is given by:

Theorem 4.1.3 Suppose that $\left(\mathbb{Y}_{t}, t \geq 0\right)$ satisfies Eq. (1) and has finite second moments for $\boldsymbol{\theta} \in \widetilde{\boldsymbol{\Theta}}$. In addition, suppose that $E\left[\exp \left(\boldsymbol{\theta}^{\top} \mathbb{H}_{t}\right)\right]<\infty$ for $t$ large enough. Then, the MLE $\widehat{\boldsymbol{\theta}}_{t}$ satisfies under $P_{\mathbb{Y}}$

$$
[\mathbb{H}]_{t}^{1 / 2} \cdot\left(\widehat{\boldsymbol{\theta}}_{t}-\boldsymbol{\theta}\right) \stackrel{\mathcal{D}}{\longrightarrow} \mathcal{N}\left(\mathbf{0}, I_{2 \times 2}\right), \text { as } t \rightarrow \infty .
$$

Moreover, $\widehat{\boldsymbol{\theta}}_{t}$ is efficient in the sense of Hájek-Le Cam's convolution theorem.

Proof See Appendix A.7.

Corollary 4.1.4 Under the same assumptions as in Theorem 4.1.3, one obtains

$$
t^{1 / 2}\left(\widehat{\boldsymbol{\theta}}_{t}-\boldsymbol{\theta}\right) \stackrel{\mathcal{D}}{\longrightarrow} \mathcal{N}\left(\mathbf{0}_{2}, \boldsymbol{G}_{\infty}^{-1}\right), \text { as } t \rightarrow \infty,
$$

where

$$
\boldsymbol{G}_{\infty}:=\left(\begin{array}{cc}
E\left(\left\langle\overline{\mathbf{A}} \mathbb{Y}_{\infty}, \overline{\mathbf{A}} \mathbb{Y}_{\infty}\right\rangle_{\boldsymbol{\Sigma}}\right) & E\left(\left\langle\overline{\mathbf{A}} \mathbb{Y}_{\infty}, \mathbb{Y}_{\infty}\right\rangle_{\boldsymbol{\Sigma}}\right) \\
E\left(\left\langle\overline{\mathbf{A}} \mathbb{Y}_{\infty}, \mathbb{Y}_{\infty}\right\rangle_{\boldsymbol{\Sigma}}\right) & E\left(\left\langle\mathbb{Y}_{\infty}, \mathbb{Y}_{\infty}\right\rangle_{\boldsymbol{\Sigma}}\right)
\end{array}\right)
$$

which is positive definite by Cauchy-Schwarz's inequality.

Proof Recall the result of Lemma 3.3.2; the continuous mapping theorem yields that $\left([\mathbb{H}]_{t} / t\right)^{1 / 2} \rightarrow G_{\infty}^{1 / 2} \mathrm{P}_{\mathbb{Y}}$-a.s. By application of Theorem 4.1.3 and Slutsky's lemma, the result follows directly. 


\subsection{Asymptotics for $\psi$-GrOU}

We present a decomposition of the MLE $\widehat{\psi}$ in the following lemma:

Lemma 4.2.1 In the context of Theorem 3.4.4, we have that

$$
\widehat{\boldsymbol{\psi}}_{t}-\boldsymbol{\psi}=[\mathbb{I}]_{t}^{-1} \mathbb{M}_{t}, \quad t \geq 0,
$$

where $\mathbb{M}_{t}:=\int_{0}^{t} \mathbb{Y}_{s} \otimes d \mathbb{W}_{s}$ is the (martingale) remainder vector and $[\mathbb{I}]_{t}=\mathbf{K}_{t} \otimes \mathbf{I}_{d \times d}$.

Proof See Appendix A.8.

Similarly to Sect. 4.1, we denote by $\left(\mathrm{P}_{\mathbb{Y}}^{\psi}: \psi \in \widehat{\boldsymbol{\Psi}}\right)$ the statistical models of Lévy-driven Ornstein-Uhlenbeck processes with respect to the likelihood from Proposition 3.4.2 indexed on $\widehat{\boldsymbol{\Psi}}$. We obtain the local asymptotic normality for this sequence:

Lemma 4.2.2 The family of statistical models $\left(P_{\mathbb{Y}}^{\psi}: \boldsymbol{\psi} \in \widehat{\Psi}\right)$ with respect to $\left(\mathcal{L}_{t}(\boldsymbol{\psi} ; \mathbb{Y}): \boldsymbol{\theta} \in \widehat{\boldsymbol{\Psi}}\right)$ is locally asymptotically normal.

Proof We can apply a similar argument to the proof of Lemma 4.1.2 in $\mathbb{R}^{d}$.

As presented in Theorem 4.1.3, we obtain a central limit theorem for $\boldsymbol{\psi}$-GrOU with continuous-time observations as follows:

Theorem 4.2.3 Assume Assumptions 1 and 2 hold and that Proposition 2.4.3 can be applied. In addition, suppose that $E\left[\exp \left(\boldsymbol{\psi}^{\top} \mathbf{A}_{t}^{\boldsymbol{\Sigma}}\right)\right]<\infty$ for $t$ large enough and $\boldsymbol{\psi} \in \widehat{\boldsymbol{\Psi}}$. Then, $\widehat{\boldsymbol{\psi}}_{t}$ is consistent and we obtain:

$$
t^{1 / 2}\left(\widehat{\psi}_{t}-\psi\right) \stackrel{\mathcal{D}}{\longrightarrow} \mathcal{N}\left(\mathbf{0}_{d^{2}}, E\left(\mathbb{Y}_{\infty} \mathbb{Y}_{\infty}^{\top}\right)^{-1} \otimes \Sigma\right), \text { as } t \rightarrow \infty
$$

where we recall that $E\left(\mathbb{Y}_{\infty} \mathbb{Y}_{\infty}^{\top}\right)=\int_{0}^{\infty} e^{-s \boldsymbol{Q}} \boldsymbol{\Sigma} e^{-s} \boldsymbol{Q}^{\top} d s$. Moreover, $\widehat{\boldsymbol{\psi}}_{t}$ is efficient in the sense of Hájek-Le Cam's convolution theorem.

Proof See Appendix A.9.

We have derived an essential result for a general dynamics matrix (e.g. $\left.\operatorname{vec}^{-1}(\boldsymbol{\psi})\right)$ with diagonal elements dominating the average off-diagonal parameters row-wise. We extend the result to include the network topology and derive a corollary for such a graph-constrained estimator as follows:

Corollary 4.2.4 In the same setting as in Theorem 4.2.3, we have:

$t^{1 / 2}\left\{\operatorname{vec}\left[\boldsymbol{Q}\left(\widetilde{\boldsymbol{\psi}}_{t}\right)\right]-\operatorname{vec}[\boldsymbol{Q}(\boldsymbol{\psi})]\right\} \stackrel{\mathcal{D}}{\longrightarrow} \mathcal{N}\left(\mathbf{0}_{d^{2}}, \mathbf{D}_{\mathbf{A}} \cdot E\left(\mathbb{Y}_{\infty} \mathbb{Y}_{\infty}^{\top}\right)^{-1} \otimes \boldsymbol{\Sigma} \cdot \mathbf{D}_{\mathbf{A}}\right), \quad$ as $t \rightarrow \infty$, where $\mathbf{D}_{\mathbf{A}}:=\operatorname{diag}\left(\operatorname{vec}\left(\mathbf{I}_{d \times d}+\overline{\mathbf{A}}\right)\right)$.

Proof By a property of the Hadamard product, observe that vec $(\mathbf{Q}(\boldsymbol{\psi}))=\operatorname{vec}\left(\mathbf{I}_{d \times d}+\overline{\mathbf{A}}\right) \odot$ $\boldsymbol{\psi}=\operatorname{diag}\left(\operatorname{vec}\left(\mathbf{I}_{d \times d}+\overline{\mathbf{A}}\right)\right) \cdot \boldsymbol{\psi}=\mathbf{D}_{\mathbf{A}} \cdot \boldsymbol{\psi}$. By Theorem 4.2.3, the result follows directly.

The term $\mathbf{D}_{\mathbf{A}}$ highlights the application of the network topology and yields a generalised form of the two-dimensional central limit theorem given in Corollary 4.1.4. 
Remark 4.2.5 Fasen (2013) proved a similar central limit theorem but for the regression on $e^{-\mathbf{Q}}$ itself which remains an alternative to the MLE approach, but the identifiability issues mentioned below Remark 2.4.4, in Sect. 2.4 hinders the direct estimation of $\boldsymbol{Q}$ from $e^{-\mathbf{Q}}$.

Until this point, we have assumed the availability of the adjacency matrix. However, sparse stochastic processes have become increasingly influential to handle high-dimension problems (Belomestny et al. 2019; Gaïffas and Matulewicz 2019; Ma et al. 2021). Regularisation through a penalty on the model parameters is an important component of this literature and we show in the next section that general Lévy-driven OU processes can be consistently transformed into GrOU processes in this context.

\section{Asymptotic theory of the Adaptive Lasso regularisation}

A key limitation of the MLEs is that the adjacency matrix should be fully specified: this limits the applicability of the GrOU process to datasets where the graph topology is known (as in Assumption 1). Also, regularisation techniques are a powerful tool to create sparse graph-like structure for high-dimensional problems (Chen et al. 2020; Ma et al. 2021). To prove that such tools can be used in our setting, we propose an Adaptive Lasso scheme and show its consistency and asymptotic normality.

\subsection{Adaptive Lasso regularisation}

Applying an $L^{1}$-penalty on the dynamics matrix $\mathbf{Q}$ to the log-likelihood, called a Lasso regression, is a common practice to introduce sparsity into $\mathbf{Q}$. Then, the regularised process can be interpreted as a proper GrOU process. In addition, a parameter allows the practitioners to tune how sparse the then-estimated adjacency matrix should be.

Definition 3 The support of a vector $\mathbb{X} \in \mathbb{R}^{d}$ (resp. a matrix $\left.\mathbf{X} \in \mathcal{M}_{d}(\mathbb{R})\right)$, denoted $\operatorname{supp}(\mathbb{Y})$ $($ resp. $\operatorname{supp}(\mathbf{X}))$, is defined as the set of indices of non-null coordinates, i.e. $\operatorname{supp}(\mathbb{X}):=\{i \in$ $\left.\{1, \ldots, d\}: \mathbb{Y}_{i} \neq 0\right\}\left(\right.$ resp. $\left.\operatorname{supp}(\mathbf{X}):=\left\{(i, j) \in\{1, \ldots, d\}^{2}: \mathrm{X}_{i j} \neq 0\right\}\right)$.

Notation 5.1.1 For a set of indices $\mathcal{I} \subseteq\{1, \ldots, d\}$ (resp. $\left.\mathcal{I} \subseteq\{1, \ldots, d\}^{2}\right)$, the restriction of $\mathbb{X}($ resp. $\mathbf{X})$ to the indices in $\mathcal{I}$ is denoted $\mathbb{X}_{\mid \mathcal{I}}$ (resp. $\left.\mathbf{X}_{\mid \mathcal{I}}\right)$.

Similarly, Adaptive Lasso (AL) regularisation schemes leverages a penalty that takes into account any $t^{1 / 2}$-consistent estimator-the MLE herein-to provide better theoretical guarantees such as consistency in variable selection (Bühlmann and Van De Geer 2011, Section 2.6) or asymptotic normality. The former is defined as the support of the estimator converging to the support of the true parameter asymptotically.

\subsection{Definition}

An AL scheme (Gaïffas and Matulewicz 2019) applied on a Lévy-driven OU process $\mathbb{Y}$ with unknown dynamics matrix $\mathbf{Q}_{0}$ yields a GrOU-like process with non-trivial adjacency matrix A. It is defined by

$$
\widehat{\mathbf{Q}}_{\mathrm{AL}, t}:=\underset{\mathbf{Q}}{\arg \max } \ell_{t}(\mathbf{Q})-\lambda\left\|\mathbf{Q} \odot\left|\widehat{\mathbf{Q}}_{t}\right|^{-\gamma}\right\|_{1},
$$


for fixed parameters $\lambda \geq 0$ and $\gamma>0$. Also, $\odot$ denotes the Hadamard product and the denominator of the penalty $\left|\widehat{\mathbf{Q}}_{t}\right|^{-\gamma}$ is evaluated elementwise. The log-likelihood is given by

$$
\ell_{t}(\mathbf{Q})=-\int_{0}^{t}\left\langle\mathbf{Q} \mathbb{Y}_{s}, d \mathbb{Y}_{s}^{c}\right\rangle_{\Sigma}-\frac{1}{2} \int_{0}^{t}\left\langle\mathbf{Q} \mathbb{Y}_{s}, \mathbf{Q} \mathbb{Y}_{s}\right\rangle_{\Sigma} d s
$$

with the corresponding $d \times d$ MLE matrix

$$
\widehat{\mathbf{Q}}_{t}:=-\mathbf{K}_{t}^{-1} \cdot \int_{0}^{t} \mathbb{Y}_{s} \cdot\left(d \mathbb{Y}_{s}^{c}\right)^{\top} .
$$

The MLE components are almost-surely non-zero and penalise more the entries that are expected to be zero.

Conditional on the knowledge of $\mathbf{Q}_{0}$, we show two oracle properties: (a) the scheme is consistent in variable selection: i.e. the support of $\widehat{\mathbf{Q}}_{\mathrm{AL}, t}$ converges to the support of the true parameter $\mathbf{Q}_{0}$ as $t \rightarrow \infty$; (b) the estimator is asymptotically normal as $t \rightarrow \infty$ over the support of the true parameter. For instance, a Lasso regression with Gaussian noise is not consistent (Zou 2006).

\subsection{Asymptotic properties}

The parameter $\lambda$ is implicitly a function of the time horizon $t$, i.e. $\lambda=\lambda(t)$. We present an equivalent to Th. 4, Gaïffas and Matulewicz (2019) for Lévy-driven OU processes.

Theorem 5.3.1 (Adapted from Th. 4, Gaïffas and Matulewicz (2019)) Suppose that Assumptions 1 and 2 hold for a Lévy-driven OU process $\mathbb{Y}$ with a true but unknown dynamics matrix $\mathbf{Q}_{0}$. For a fixed $\gamma>0$, assume that $\lambda=\lambda(t)$ satisfies $\lambda(t) t^{1 / 2} \rightarrow 0$ and $\lambda(t) t^{(1+\gamma) / 2} \rightarrow \infty$ as $t \rightarrow \infty$. Then, under the assumption that $\mathbf{Q}_{0}$ is known, we obtain:

1. Consistency of the variable selection: $P\left(\operatorname{supp}\left(\widehat{\mathbf{Q}}_{\mathrm{AL}, t}\right)=\operatorname{supp}\left(\mathbf{Q}_{0}\right)\right) \rightarrow 1$ as $t \rightarrow \infty$.

2. Asymptotic normality:

$$
\begin{aligned}
& t^{1 / 2}\left(\operatorname{vec}\left(\widehat{\mathbf{Q}}_{\mathrm{AL}, t}\right)-\operatorname{vec}\left(\mathbf{Q}_{0}\right)\right)_{\mid \mathcal{Q}_{0}} \stackrel{\mathcal{D}}{\longrightarrow} \mathcal{N}\left(\mathbf{0}_{d^{2}}, E\left(\mathbb{Y}_{\infty} \mathbb{Y}_{\infty}^{\top}\right)_{\mid \mathcal{Q}_{0} \times \mathcal{Q}_{0}}^{-1} \otimes \boldsymbol{\Sigma}_{\mid \mathcal{Q}_{0} \times \mathcal{Q}_{0}}\right), \text { as } t \rightarrow \infty, \\
& \text { where } \mathcal{Q}_{0}:=\operatorname{supp}\left(\mathbf{Q}_{0}\right) .
\end{aligned}
$$

Proof See Appendix A.10.

Note that the adjacency matrix can therefore be estimated as follows:

$$
\left(\widehat{\mathbf{A}}_{\mathrm{AL}, t}\right)_{i j}:=\mathbb{I}_{\{x \neq 0\}}\left(\left(\widehat{\mathbf{Q}}_{\mathrm{AL}, t}\right)_{i j}\right),
$$

and the $\boldsymbol{\theta}$-GrOU inference can be applied next as a simplification step for high-dimensional applications although the impact of model misspecification is left for future research. Finally, the penalty parameter $\lambda$ can be chosen to reach a given sparsity criterion (trial-and-error) or by cross-validation (Gaïfas and Matulewicz 2019, Section 4.1).

\section{An extension to a volatility-modulated GrOU process}

Stationary noise distributions are usually too simplistic to explain the intrinsic variability of the data. Volatility modulation adds a stochastic scaling factor (Belomestny et al. 2019; 
Cai et al. 2016) which follows its own dynamics to better represent exogenous source of uncertainty (Pigorsch and Stelzer 2009b; Yang et al. 2020) whilst a jump component helps to model unforeseen perturbations or rare calendar events (Barndorff-Nielsen and Veraart 2012).

We extend the framework of Sect. 2 to include a stochastic volatility modulation through a positive semidefinite Ornstein-Uhlenbeck (PSOU hereafter) process (Pigorsch and Stelzer 2009a, b) and a time-changed jump term.

For the latter term, we adapt the univariate framework introduced in Barndorff-Nielsen and Veraart (2012) to the multivariate case. We find that the volatility modulation and the jump term preserve the core properties of the model-i.e. its stationarity and ergodicity-which in turn imply that extensions of the results from Sects. 3 and 4 hold.

Notation 6.0.1 For a process $\left(\mathbb{X}_{t}, t \geq 0\right) \subseteq \mathbb{R}^{d}$, we denote by $\varphi_{\mathbb{X}_{t}}(\boldsymbol{u}):=E\left[\exp \left(i \boldsymbol{u}^{\top} \mathbb{X}_{t}\right)\right]$ its characteristic function at time $t$. Similarly, for an $\mathcal{M}_{d}(\mathbb{R})$-valued process $\left(\boldsymbol{X}_{t}\right)$, we write $\varphi_{\boldsymbol{X}_{t}}(\boldsymbol{u}):=E\left\{\exp \left[i \operatorname{tr}\left(\boldsymbol{u}^{\top} \boldsymbol{X}_{t}\right)\right]\right\}$. Finally, we denote by $\log \varphi(\cdot)$ the distinguished logarithm of $\varphi$ for an infinitely divisible distribution (Sato et al. 1999, Lemma 7.6)

Remark 6.0.2 For a two-sided Lévy process $\left(\boldsymbol{L}_{t}, t \in \mathbb{R}\right) \subseteq \mathcal{M}_{d}(\mathbb{R})$ and for adapted processes $\left(\boldsymbol{A}_{t}=\left(A_{i j, t}\right), t \geq 0\right),\left(\boldsymbol{B}_{t}=\left(B_{i j, t}\right), t \geq 0\right) \subseteq \mathcal{M}_{d}(\mathbb{R})$ with respect to $\boldsymbol{L}$, we denote by $\int_{0}^{t} \boldsymbol{A}_{a} d \boldsymbol{L}_{s} \boldsymbol{B}_{s}$ the matrix whose $(i, j)$-th element is given by $\sum_{k, l} \int_{0}^{t} A_{i k, s} B_{l j, s} d L_{k l, s}$.

\subsection{Model extension}

Consider the continuous-time process $\left(\mathbb{Y}_{t}^{(v)}, t \geq 0\right)$ satisfying the stochastic differential equation

$$
d \mathbb{Y}_{t}^{(v)}=-\mathbf{Q} \mathbb{Y}_{t}^{(v)} d t+\Sigma_{t}^{1 / 2} d \mathbb{W}_{t}+d \mathbb{J}_{T_{t}}, \quad t \geq 0,
$$

where $\left(\boldsymbol{\Sigma}_{t}, t \in \mathbb{R}\right)$ is a càdlàg stochastic volatility (SV) process. In addition, ( $\left.\mathbb{W}_{t}, t \in \mathbb{R}\right)$ is a d-dimensional Brownian motion process, $\left(T_{t}, t \in \mathbb{R}\right)$ is an increasing continuous process where $T_{t} \rightarrow \pm \infty \mathrm{P}_{0}$-a.s. as $t \rightarrow \pm \infty$ and $\left(\mathbb{J}_{t}, t \in \mathbb{R}\right)$ is a two-sided pure-jump Lévy process with characteristic triplet $\left(\gamma_{\mathbb{J}}, \mathbf{0}, v_{\mathbb{J}}\right)$ with respect to the truncation function $\tau(z):=$ $\mathbb{I}_{\left\{x \in \mathbb{R}^{d}:\|x\| \leq 1\right\}}(z)$ (see Sect. 2.2).

Under standard regularity conditions (see Sects. 6.3.3 and 6.4), we know that the unique candidate for a stationary solution to Eq. (11) is

$$
\mathbb{Y}_{t}^{(v)}=\int_{-\infty}^{t} e^{-(t-s) \mathbf{Q}} \Sigma_{s}^{1 / 2} d \mathbb{W}_{s}+\int_{-\infty}^{t} e^{-(t-s) \mathbf{Q}} d \mathbb{J}_{T_{s}}, \quad t \in \mathbb{R},
$$

where both terms are well-defined by Corollary 4.1, Basse-O'Connor et al. (2014) (see Sects. 6.3.2 and 6.4.2). Note that we have now extended the domain from $t \geq 0$ to $t \in \mathbb{R}$ (Brockwell 2009, Remark 1). We study each term separately in Sects. 6.3 and 6.4.

Regarding the volatility process, consider a positive definite matrix $\boldsymbol{V} \in \mathbb{S}_{d}^{++}$and a two-sided $d \times d$ matrix Lévy subordinator $\left(\boldsymbol{L}_{t}, t \in \mathbb{R}\right.$ ) (Barndorff-Nielsen and Pérez-Abreu 2008) such that $\left(\Sigma_{t}, t \in \mathbb{R}\right)$ is a stationary positive semidefinite Ornstein-Uhlenbeck (PSOU) process (Pigorsch and Stelzer 2009b), i.e. given by

$$
\boldsymbol{\Sigma}_{t}=\int_{-\infty}^{t} e^{-(t-s) \boldsymbol{V}} d \boldsymbol{L}_{s} e^{-(t-s) \boldsymbol{V}^{\top}}, \quad t \in \mathbb{R} .
$$

We recall the existence conditions of this stationary process in Sect. 6.3.1. 
Assumption 3 We assume the independence between $\mathbb{W}, \boldsymbol{L}, \mathbb{J}$ and $T$.

In the following two subsections, we characterise both terms presented in the stationary solution in Eq. (12). We then prove that the resulting process $\left(\mathbb{Y}_{t}^{(v)}\right)$ is mixing hence ergodic which requires additional definitions presented in the next section.

To prove the ergodicity of the model presented in Sect. 6.1, we augment our framework with another class of stochastic mixed moving average (MMA) processes which have wellstudied asymptotic behaviour such as the mixing and ergodic properties (see Sect. 6.2).

\subsection{Lévy bases, MMA processes and the mixing property}

In this section, we recall the definitions of Lévy bases, characteristic quadruplet and Lévydriven MMA processes.

Definition 4 (Fuchs and Stelzer 2013, Definition 3.1) A $d$-dimensional Lévy basis on $S \times \mathbb{R}$ is an $\mathbb{R}^{d}$-valued random measure $\Lambda=\left\{\Lambda(B): B \in \mathcal{B}_{b}(S \times \mathbb{R})\right\}$ satisfying:

(a) the distribution of $\Lambda(B)$ is infinitely divisible for all $B \in \mathcal{B}_{b}(S \times \mathbb{R})$;

(b) for any $n \in \mathbb{N}$ and pairwise disjoint sets $B_{1}, \ldots, B_{n} \in \mathcal{B}_{b}(S \times \mathbb{R})$ the random variables $\Lambda\left(B_{1}\right), \ldots, \Lambda\left(B_{n}\right)$ are independent and

(c) for any pairwise disjoint sets $\left(B_{i} \in \mathcal{B}_{b}(S \times \mathbb{R})\right.$, $\left.i \in \mathbb{N}\right)$ satisfying $\bigcup_{n \in \mathbb{N}} B_{n} \in$ $\mathcal{B}_{b}(S \times \mathbb{R})$ the series $\sum_{n=1}^{\infty} \Lambda\left(B_{n}\right)$ converges almost surely and it holds that $\Lambda\left(\bigcup_{n \in \mathbb{N}}\right)=$ $\sum_{n \in \mathbb{N}} \Lambda\left(B_{n}\right)$ almost surely.

Remark 6.2.1 Here, we take $S=\mathbb{S}_{d}^{++}$and note that a definition of $\mathbb{S}_{d}^{+}$-valued Lévy bases on $S \times \mathbb{R}$ would be formulated similarly.

As in Barndorff-Nielsen and Stelzer (2011); Fuchs and Stelzer (2013), we restrict ourselves to time-homogeneous and factorisable Lévy bases, i.e. with characteristic function

$$
\mathrm{E}\left[\exp \left(i z^{\top} \Lambda(B)\right)\right]=\exp (\log \varphi(z) \times \Pi(B)), \quad \text { for any } B \in \mathcal{B}_{b}\left(\mathbb{S}_{d}^{++} \times \mathbb{R}\right),
$$

where $\Pi=\pi \otimes \lambda^{l e b}$ is the product of a probability measure $\pi$ on $\mathbb{S}_{d}^{++}$and the Lesbesgue measure on $\mathbb{R}$ and $z \mapsto \varphi(z)$ is the characteristic function of an infinitely divisible distribution (Sect. 2.2) characterised, say, by a triplet $(\gamma, \Sigma, v)$.

Let $\tilde{\gamma}(A):=\gamma$ and $\widetilde{\boldsymbol{\Sigma}}(A):=\Sigma$ be trivial maps from $\mathbb{S}_{d}^{++}$to, respectively, $\mathbb{R}^{d}$ and $\mathbb{S}_{d}^{+}$, and let $\widetilde{v}(d x, A):=v(d x)$ be an extension of $v$ to $\mathbb{R}^{d} \times \mathbb{S}_{d}^{++}$. As per Section 3 , Fuchs and Stelzer (2013) and p. 162 Barndorff-Nielsen et al. (2018), any such quadruplet $(\widetilde{\gamma}, \widetilde{\Sigma}, \widetilde{v}, \pi)$ characterises completely in law a Lévy basis $\Lambda$ in the sense of Definition 33, BarndorffNielsen et al. (2018) where $\pi$ is then called the intensity measure (as an extension of the control measure from Rajput and Rosinski (1989)). Indeed, $S \mapsto \int_{S} \tilde{\gamma}(A) \pi(d A)=\gamma \pi(S)$ and $S \mapsto \int_{S} \widetilde{\Sigma}(A) \pi(d A)=\Sigma \pi(S)$ are respectively signed and unsigned measures on $\left(\mathbb{S}_{d}^{++}, \mathcal{B}\left(\mathbb{S}_{d}^{++}\right)\right)$; while $\int_{S} v(d x, A) \pi(d A)=v(d x) \pi(S)$ is a Lévy measure on $\mathbb{R}$ for a fixed $S \in \mathbb{S}_{d}^{++}$.

For the existence of integrals with respect to a Lévy basis, see Th. 3.2, Fuchs and Stelzer (2013) and Th. 2.7, Rajput and Rosinski (1989). We recall the definition of multivariate MMA processes as follows:

Definition 5 (Fuchs and Stelzer 2013, adapted from Definition 3.3) 
Let $\Lambda$ be an $\mathbb{R}^{d}$-valued Lévy basis on $S \times \mathbb{R}$ and let $f: \S \times \mathbb{R} \rightarrow \mathcal{M}_{n, d}(\mathbb{R})$ be a measurable function. If the process

$$
\int_{S} \int_{\mathbb{R}} f(A, t-s) \Lambda(d A, d s)
$$

exists in the sense of Theorem 3.2, Fuchs and Stelzer (2013), for all $t \in \mathbb{R}$, it is called an $n$-dimensional mixed moving average process (MMA for short). The function $f$ is said to be its kernel function.

Finally, we also recall the definition of mixing processes:

Definition 6 A process $\left(\mathbb{Y}_{t}, t \in \mathbb{R}\right)$ is mixing if and only if, for any $t \in \mathbb{R}$

$$
\mathrm{P}\left(\left\{\mathbb{Y}_{t} \in A\right\} \cap\left\{\mathbb{Y}_{t+h} \in B\right\}\right) \longrightarrow \mathrm{P}\left(\mathbb{Y}_{t} \in A\right) \mathrm{P}\left(\mathbb{Y}_{t+h} \in B\right), \quad \text { as } h \rightarrow \infty,
$$

for any $A \in \mathcal{F}_{-\infty}^{t}=\sigma\left(\left\{\mathbb{Y}_{s}, s \leq t\right\}\right), B \in \mathcal{F}_{t+h}^{\infty}=\sigma\left(\left\{\mathbb{Y}_{s}, s \geq t+h\right\}\right)$.

It is straightforward to observe that this implies ergodicity. Fuchs and Stelzer (2013) adapt the mixing conditions given in Maruyama (1970) and Rosiński and Żak (1997) to the multivariate context and prove that Lévy-driven MMA processes are mixing (Theorem 3.5 therein).

\subsection{Stochastic volatility component}

This PSOU process and several extensions have been developed and studied in the last decade (Barndorff-Nielsen and Stelzer 2011; Barndorff-Nielsen and Veraart 2012; Fuchs and Stelzer 2013; Pigorsch and Stelzer 2009a,b). The ability to model specific marginal distributions whilst remaining tractable gives a flexible and powerful method to augment our original model (Pigorsch and Stelzer 2009b, Sections 4.2 and 5).

Denote by $\rho: \mathbb{S}_{d} \rightarrow \mathbb{S}_{d}$ the linear operator $\boldsymbol{X} \mapsto \boldsymbol{V} \boldsymbol{X}+\boldsymbol{X} \boldsymbol{V}^{\top}$ such that $e^{t \rho}\left(\mathbb{S}_{d}\right)=\mathbb{S}_{d}$ (Pigorsch and Stelzer 2009b, Section 3).

\subsubsection{Invariant distribution and operator self-decomposability}

The literature focuses on the existence and uniqueness of the invariant distribution given in Eq. (13) (Masuda 2004; Pigorsch and Stelzer 2009b). Suppose that

$$
\int_{\mathbb{S}_{d}^{+}}(\log \|\boldsymbol{Z}\| \vee 0) \nu_{\boldsymbol{L}}(d \boldsymbol{Z})<\infty,
$$

then, there exists a unique invariant distribution $F_{\Sigma}$ (according to Prop. 2.2, Masuda (2004), and Th. $4.1 \&$ 4.2, Sato and Yamazato (1984)) which we take in its matrix-valued form. The distribution $F_{\Sigma}$ is operator self-decomposable with respect to the linear operator $\rho$ (Pigorsch and Stelzer 2009b, Prop. 4.3). Hence, $F_{\Sigma}$ is absolutely continuous if the support of $\boldsymbol{L}$ is nondegenerate (i.e. $v_{L}(a+S)<1$ for any $a \in \mathbb{S}_{d}$ and $S \subseteq \mathbb{S}_{d} \operatorname{such}$ that $\operatorname{dim}(S) \leq \operatorname{dim}\left(\mathbb{S}_{d}\right)-1$, see Yamazato (1983)). In that case, note that this stationary distribution is almost surely concentrated on $\mathbb{S}_{d}^{++}$with respect to the Lebesgue measure (Pigorsch and Stelzer 2009b, Th. 4.4). According to Sect. 2.4, Pigorsch and Stelzer (2009b), one can write for $t \geq 0$ that

$$
\boldsymbol{\Sigma}_{t}=e^{-t \boldsymbol{V}} \boldsymbol{\Sigma}_{0} e^{-t \boldsymbol{V}^{\top}}+\int_{0}^{t} e^{-(t-s) \boldsymbol{V}} d \boldsymbol{L}_{s} e^{-(t-s) \boldsymbol{V}^{\top}},
$$


or, in vectorised form, that

$$
\operatorname{vec}\left(\boldsymbol{\Sigma}_{t}\right)=e^{-t\left(\boldsymbol{V} \otimes \mathbf{I}_{d \times d}+\mathbf{I}_{d \times d} \otimes \boldsymbol{V}\right)} \operatorname{vec}\left(\boldsymbol{\Sigma}_{0}\right)+\int_{0}^{t} e^{-(t-s)\left(\boldsymbol{V} \otimes \mathbf{I}_{d \times d}+\mathbf{I}_{d \times d} \otimes \boldsymbol{V}\right)} d \operatorname{vec}\left(\boldsymbol{L}_{s}\right) .
$$

Note that if $\boldsymbol{V} \in \mathbb{S}_{d}^{++}$then $\boldsymbol{V} \otimes \mathbf{I}_{d \times d}+\mathbf{I}_{d \times d} \otimes \boldsymbol{V} \in \mathbb{S}_{d}^{++}$. In particular, $\left(\boldsymbol{\Sigma}_{t}, t \geq 0\right)$ satisfies

$$
d \boldsymbol{\Sigma}_{t}=-\left(\boldsymbol{V} \boldsymbol{\Sigma}_{t-}+\boldsymbol{\Sigma}_{t-} \boldsymbol{V}^{\top}\right) d t+d \boldsymbol{L}_{t}, \quad t \geq 0,
$$

where $\Sigma_{0} \in \mathbb{S}_{d}^{+}$. We conclude that $\left(\boldsymbol{\Sigma}_{t}\right)$ is an MMA process (Fuchs and Stelzer 2013, Def. 3.3).

Proposition 6.3.1 Suppose the framework given in Sects. 6.1 and 6.3 holds. Then, $\left(\Sigma_{t}, t \in \mathbb{R}\right)$ is an MMA process as given in Definition 5.

Proof See Appendix A.11.

\subsubsection{Characteristic function}

Recall that $\left(\boldsymbol{L}_{t}, t \in \mathbb{R}\right)$ is taken to be a two-sided matrix Lévy subordinator process: a process that is $\mathbb{S}_{d}^{+}$-increasing (such that $\boldsymbol{L}_{t}-\boldsymbol{L}_{s} \in \mathbb{S}_{d}^{+}$for any $t>s$ ) and of finite variation (Barndorff-Nielsen and Stelzer 2007).

It is characterised by a triplet $\left(\boldsymbol{\gamma}_{\boldsymbol{L}}, \mathbf{0}, v_{\boldsymbol{L}}\right)$ where $\boldsymbol{\gamma}_{\boldsymbol{L}} \in \mathbb{S}_{d}^{+}$and $\nu_{L}$ is a Lévy measure on the space of positive semidefinite matrices $\mathbb{S}_{d}^{+}$such that

$$
\int_{\mathbb{S}_{d}^{+}}(\|\boldsymbol{Z}\| \wedge 1) v_{\boldsymbol{L}}(d \boldsymbol{Z})<\infty, \quad \text { and } \quad v_{\boldsymbol{L}}(\{\mathbf{0}\})=0 .
$$

According to Part 1, Barndorff-Nielsen and Shiryaev (2015), given Eq. (16), its characteristic function at time $t \in \mathbb{R}$ given by

$$
\varphi_{\boldsymbol{L}_{t}}(\boldsymbol{U}):=\exp \left\{t\left[i \operatorname{tr}\left(\boldsymbol{\gamma}_{\boldsymbol{L}} \boldsymbol{Z}\right)+\int_{\mathbb{S}_{d}^{+} \backslash\{\boldsymbol{0}\}}\left(e^{i \operatorname{tr}(\boldsymbol{Z U})}-1\right) v_{\boldsymbol{L}}(d \boldsymbol{Z})\right]\right\}, \quad \text { for } \boldsymbol{U} \in \mathbb{S}_{d},
$$

with respect to the truncation function $\tilde{\tau}(\boldsymbol{X}) \equiv 0$ on $\mathcal{M}_{d}(\mathbb{R})$ (Barndorff-Nielsen and Shiryaev 2015, Part 1).

Theorem 4.9, Pigorsch and Stelzer (2009b) yields that if $\boldsymbol{V} \in \mathbb{S}_{d}^{++}$and Eqs. (14) and (16) hold, then the PSOU process $\left(\boldsymbol{\Sigma}_{t}, t \in \mathbb{R}\right)$ is strictly stationary and its distribution is infinitely divisible with characteristic function

$$
\varphi_{\boldsymbol{\Sigma}}(\boldsymbol{U})=\exp \left\{i \operatorname{tr}\left(\boldsymbol{\gamma}_{\boldsymbol{\Sigma}}\right)+\int_{\mathbb{S}_{d}^{+} \backslash\{\boldsymbol{0}\}}\left(e^{i \operatorname{tr}(\boldsymbol{Z U})}-1\right) v_{\boldsymbol{\Sigma}}(d \boldsymbol{Z})\right\}, \quad \text { for } \boldsymbol{U} \in \mathbb{S}_{d},
$$

where $\gamma_{\Sigma}:=\rho^{-1}\left(\gamma_{L}\right) \in \mathbb{S}_{d}^{+}$and

$$
v_{\boldsymbol{\Sigma}}(S):=\int_{0}^{\infty} \int_{\mathbb{S}_{d}^{+} \backslash\{\boldsymbol{0}\}} \mathbb{I}_{S}\left(e^{-s \boldsymbol{V}} \boldsymbol{Z} e^{-s \boldsymbol{V}^{\top}}\right) \nu_{\boldsymbol{L}}(d \boldsymbol{Z}) d s, \quad \text { for } S \in \mathcal{B}\left(\mathbb{S}_{d}^{+} \backslash\{\mathbf{0}\}\right) .
$$

In that case, note that $v_{\Sigma}\left(\mathbb{S}_{d} \backslash \mathbb{S}_{d}^{+}\right)=0$.

Following the characterisation of $\left(\Sigma_{t}, t \in \mathbb{R}\right)$, we present the second part of the noise in Eq. (11) which is a pure-jump time-changed Lévy process. 


\subsubsection{Stochastic volatility of a multivariate OU process}

Suppose that $\left(\Sigma_{t}\right)$ is strictly stationary. Let $a<b \in \mathbb{R} \cup\{ \pm \infty\}$ and consider the process

$$
\mathbb{F}_{a b}^{(1)}=\int_{a}^{b} e^{-(b-s) \mathbf{Q}} \Sigma_{s}^{1 / 2} d \mathbb{W}_{s} .
$$

By Proposition 6.3.1, $\left(\boldsymbol{\Sigma}_{t}\right)$ is an Lévy-driven MMA process hence locally uniformly bounded as given by Theorem 4.3, (ii), Barndorff-Nielsen and Stelzer (2011). Therefore, for any $t \geq 0$, the integral

$$
\int_{-\infty}^{t} e^{-(t-s) \mathbf{Q}} \Sigma_{s} e^{-(t-s) \mathbf{Q}^{\top}} d s \text { is a Lebesgue integral of }\left(\boldsymbol{\Sigma}_{t}\right) \omega \text {-wise. }
$$

We obtain the following distributional property for $F_{a b}^{(1)}$ :

Proposition 6.3.2 The distribution of $\mathbb{F}_{a b}^{(1)}$ is non-degenerate in the sense of Yamazato (1983) for any $a<b \in \mathbb{R} \cup\{ \pm \infty\}$

Proof See Appendix A.12.

In the case when $a=-\infty$, we can prove the stationarity of the stochastic volatility term as follows

Proposition 6.3.3 If $\left(\Sigma_{t}, t \in \mathbb{R}\right)$ is strictly stationary, then $\left(\mathbb{F}_{-\infty t}^{(1)}, t \in \mathbb{R}\right)$ is strictly stationary.

Proof See Appendix A.13.

\subsection{Pure-jump component}

Let us next consider the pure-jump process $\left(\mathbb{J}_{T_{t}}, t \in \mathbb{R}\right)$. Suppose that

$$
\int_{\mathbb{R}^{d}}(\log \|z\| \vee 0) \nu_{\mathbb{J}}(d z)<\infty,
$$

as well as

$$
\int_{\mathbb{R}^{d}}\left(\|z\|^{2} \wedge 1\right) v_{\mathbb{J}}(d z)<\infty
$$

\subsubsection{Characteristic function}

Since $\mathbb{J}$ is a pure-jump Lévy process and given Eq. (20), we have

$$
\varphi_{\mathbb{J}_{1}}(\boldsymbol{u})=\exp \left\{i \boldsymbol{u}^{\top} \gamma_{\mathbb{J}}+\int_{\mathbb{R}^{d} \backslash\{\boldsymbol{0}\}}\left(e^{i \boldsymbol{u}^{\top} z}-1-i \boldsymbol{u}^{\top} \boldsymbol{z} \tau(\boldsymbol{z})\right) d \nu_{\mathbb{J}}(d \boldsymbol{z})\right\} .
$$

We recall that a stochastic process $X$ is adapted with respect to $T$ if $X$ is constant on any interval $\left[T_{t-}, T_{t}\right]$ for any $t \in \mathbb{R}$. According to Lemma 10.14, Jacod (1979), since $T_{-t} \rightarrow-\infty$ as $t \rightarrow-\infty$ and $T$ is continuous, then $\mathbb{J}$ is $T$-adapted. Similarly to Section 1.2.2, BarndorffNielsen and Veraart (2012), all the base properties of $\mathbb{J}$ carry over to the time-changed process. Therefore, the characteristic function of $\left(\mathbb{J}_{T_{t}}\right)$ is given by

$$
\varphi_{\mathbb{J}_{T_{t}}}(\boldsymbol{u})=\exp \left\{T_{t} \cdot \log \varphi_{\mathbb{J}_{1}}(\boldsymbol{u})\right\} .
$$

This implies that $\left(\mathbb{J}_{T_{t}}, t \in \mathbb{R}\right)$ has a characteristic triplet $\left(T \gamma_{\mathfrak{J}}, \mathbf{0}, T \otimes v_{\mathbb{J}}\right)$. 


\subsubsection{Integrated time-changed pure-jump process}

Let $a<b \in \mathbb{R} \cup\{ \pm \infty\}$ and consider the process

$$
\mathbb{F}_{a b}^{(2)}:=\int_{a}^{b} e^{-(b-s) \mathbf{Q}} d \mathbb{J}_{T_{s}} .
$$

Consider the case where $a<b \in \mathbb{R} \cup\{ \pm \infty\}$. According to Corollary 4.1, Basse-O'Connor et al. (2014), this integral in well-defined since $\left\|e^{-s \mathbf{Q}} \boldsymbol{x}\right\| \leq\|\boldsymbol{x}\|$ for any $s \geq 0$ and $\int_{\mathbb{R}^{d}}\left(\|z\|^{2} \wedge\right.$ 1) $v_{\mathbb{J}}(d z)<\infty$ from Eq. (20).

Conditional on the knowledge of $T$ and by independence between $T$ and $\mathbb{J}$, Lemma 15.1, p. 496, Cont and Tankov (2004) yields

$$
\begin{aligned}
\mathrm{E}\left[\exp \left(i \boldsymbol{u}^{\top} \mathbb{F}_{a t}^{(2)}\right) \mid T\right] & =\mathrm{E}\left[\operatorname { e x p } \left(\int_{a}^{t} i \boldsymbol{u}^{\top} e^{\left.\left.-(t-s) \mathbf{Q}_{d} \mathbb{J}_{T_{s}}\right) \mid T\right]}\right.\right. \\
& =\exp \left[\int_{a}^{t} \log \varphi_{\mathbb{J}_{1}}\left(e^{-(t-s) \mathbf{Q}^{\top}} \boldsymbol{u}\right) d T_{s}\right] .
\end{aligned}
$$

Additionally, $\mathbb{F}^{(2)}$ has a characteristic triplet with drift

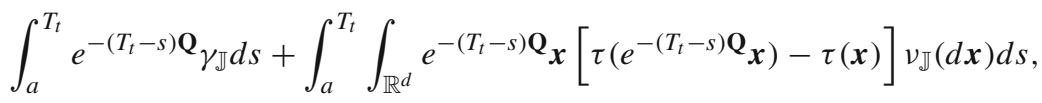

and Lévy measure

$$
\int_{a}^{T_{t}} \int_{\mathbb{R}^{d} \backslash\{\mathbf{0}\}} \mathbb{I}_{E}\left(e^{\left(T_{t}-s\right) \mathbf{Q}_{\boldsymbol{x}}}\right) v_{\mathbb{J}}(d \boldsymbol{x}) d s, \quad E \in \mathcal{B}\left(\mathbb{R}^{d}\right),
$$

by Lemma 3, Kallsen and Shiraev (2002).

Proposition 6.4.1 $\left(\mathbb{F}_{-\infty t}^{(2)}, t \in \mathbb{R}\right)$ is strictly stationary.

Proof The statement can be proved similarly to Proposition 6.3 .3 since $\left(T_{t}\right)$ is almost surely increasing which is not repeated here for the sake of brevity.

Proposition 6.4.2 Suppose the framework given in Sects. 6.1, 6.3 and 6.4 holds. Then, $\left(\mathbb{F}_{-\infty t}^{(2)}, t \in \mathbb{R}\right)$ is a Lévy-driven MMA process hence mixing.

Proof See Appendix A.14.

Proposition 6.4.2 is important since the time-changed pure-jump component does not benefit from the Gaussian structure of $\mathbb{F}^{(1)}$ and MMA processes alleviate this complication.

\subsection{Stationarity and ergodicity}

If Eqs. (14), (16) and (19) hold, then $\left(\mathbb{Y}_{t}^{(v)}, t \in \mathbb{R}\right)$ can be expressed as

$$
\mathbb{Y}_{t}^{(v)}=\mathbb{F}_{-\infty t}^{(1)}+\mathbb{F}_{-\infty t}^{(2)}, \quad t \in \mathbb{R},
$$

as given in Eq. (12). Both terms have characteristic functions given in Sects. 6.3.2 and 6.4.2. Similarly to the Lévy-driven case in Masuda (2004), Eq. (21) yields solutions which have operator self-decomposable distributions given the independence between $\boldsymbol{L}, \mathbb{W}, \mathbb{J}$ and $T$. Indeed, we write for $t_{2}>t_{1} \in \mathbb{R}$

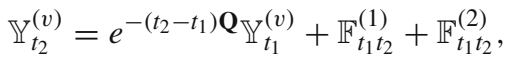


where the three terms on the right-hand side are independent and the stationary distribution is absolutely continuous if the support of $\mathbb{J}$ is non-degenerate (see Sect. 6.3.1).

Proposition 6.5.1 Suppose the framework given in Sects. 6.1, 6.3 and 6.4. If $\left(\Sigma_{t}, t \in \mathbb{R}\right)$ is strictly stationary, then $\left(\mathbb{Y}_{t}^{(v)}, t \in \mathbb{R}\right)$ is also strictly stationary.

Proof From the stationarity of both right-hand side terms of Eq. (21) given by Propositions 6.3.3 and 6.4.1, the result follows directly.

We prove that $\left(\Sigma_{t}, t \in \mathbb{R}\right)$ and $\left(\mathbb{Y}_{t}^{(v)}, t \in \mathbb{R}\right)$ are ergodic in the following proposition:

Proposition 6.5.2 Suppose the framework given in Sects. 6.1, 6.3 and 6.4 holds. Then, $\left(\Sigma_{t}, t \in \mathbb{R}\right)$ and $\left(\mathbb{Y}_{t}^{(v)}, t \in \mathbb{R}\right)$ are mixing and hence ergodic.

Proof See Appendix A.15.

The mixing and ergodicity properties of $\left(\boldsymbol{\Sigma}_{t}\right)$ are important for statistical inference on the stochastic volatility which is outside the scope of this article. We have proved that the resulting process is well-defined, stationary and ergodic. Therefore, conditional on the stochastic volatility, the estimator central limit theorems from Sect. 4 hold under stochastic volatility modulation.

\section{Conclusion}

In this article, we tackle the problem of modelling sparse interactions between multiple time series. For this purpose, we have defined the Graph Ornstein-Uhlenbeck process-a Lévydriven Ornstein-Uhlenbeck process adapted for graph structures - of which we propose two different configurations. We first consider a network-wide parametrisation where there is only one parameter to characterise momentum across all nodes and another unique parameter for the network effect. The first estimator is robust against the curse of dimensionality whilst only providing a scarce feedback on network interactions. Then, we consider an augmented version of this estimator with node-dependent momentum parameter and a different network effect for each of a node's neighbours. We derive the well-definedness, existence, uniqueness and efficiency of those estimators and we prove three novel central limit theorems (CLT) as the time horizon goes to infinity for both MLEs and an Adaptive Lasso scheme. The CLTs are a necessary step for both hypothesis tests (Morales et al. 2000) and quantifying uncertainty in the inference of graphical and/or high-dimensional time series. Finally, we extend the GrOU process to include both a stochastic volatility and jump terms which serves as an introduction towards flexible covariance structures for graphs. We also show that the afore-mentioned properties and asymptotic behaviours hold under standard regularity and independence assumptions.

This work is the first step towards understanding the behaviour of continuous-time stochastic processes on graph structures. A limitation of the current formulation is the necessity to have a continuum of data available and recent theoretical studies and applications leverage high-frequency data sources to circumvent this issue (Brownlees et al. 2020; Kim et al. 2016). The asymptotic theory of the GrOU process in this context is presented as a preprint in Courgeau and Veraart (2020). Next, the extensions to a time-dependent and stochastic modulation of the noise open up questions on the inference of sparse Lévy-driven high-dimensional volatility or covariance structures (Belomestny et al. 2019; Cai et al. 2016; Tao et al. 2013). 
Acknowledgements The authors gratefully acknowledge the financial support from the EPSRC Centre for Doctoral Training in Financial Computing and Analytics at University College London and Imperial College London (under the grant EP/L015129/1). We also would like to thank the two anonymous reviewers and editor fortheir helpful comments on earlier drafts of the manuscript.

\section{Declarations}

Conflicts of interest On behalf of all authors, the corresponding author states that there is no conflict of interest.

Open Access This article is licensed under a Creative Commons Attribution 4.0 International License, which permits use, sharing, adaptation, distribution and reproduction in any medium or format, as long as you give appropriate credit to the original author(s) and the source, provide a link to the Creative Commons licence, and indicate if changes were made. The images or other third party material in this article are included in the article's Creative Commons licence, unless indicated otherwise in a credit line to the material. If material is not included in the article's Creative Commons licence and your intended use is not permitted by statutory regulation or exceeds the permitted use, you will need to obtain permission directly from the copyright holder. To view a copy of this licence, visit http://creativecommons.org/licenses/by/4.0/.

\section{A Proofs}

\section{A.1 Notations}

To match the framework from Küchler and Sørensen (1997), we introduce the standard notation for Jacobian and Hessian matrix as follows:

Notation A.1.1 Consider any mapping $(t, \boldsymbol{\theta}) \mapsto C_{t}(\boldsymbol{\theta})$ where $t \in(0, \infty)$ and $\boldsymbol{\theta} \in \mathbb{R}^{k}$ for some $k \in \mathbb{N}$. Define $\boldsymbol{\Theta}:=\left\{\boldsymbol{\theta} \in \mathbb{R}^{k}:\left|C_{t}(\boldsymbol{\theta})\right|<\infty, \forall t \geq 0\right\}$ such that for a fixed $t, \boldsymbol{\theta} \mapsto C_{t}(\boldsymbol{\theta})$ is a twice-differentiable mapping on int $\boldsymbol{\Theta}$. The Jacobian and the Hessian matrices with respect to $\boldsymbol{\theta}$ are denoted $\nabla C_{t}(\boldsymbol{\theta})$ and $\nabla^{2} C_{t}(\boldsymbol{\theta})$ and defined, respectively, as

$$
\nabla C_{t}(\boldsymbol{\theta}):=\left(\frac{\partial C_{t}(\boldsymbol{\theta})}{\partial \theta_{j}}, j \in\{1, \ldots, k\}\right)^{\top} \text { and } \nabla^{2} C_{t}(\boldsymbol{\theta}):=\left(\frac{\partial^{2} C(\boldsymbol{\theta})}{\partial \theta_{i} \partial \theta_{j}}, i, j \in\{1, \ldots, k\}\right),
$$

for any $\boldsymbol{\theta} \in$ int $\boldsymbol{\Theta}$.

\section{A.2 Proof of Theorem 3.3.4}

Consider the definition of a steep mapping from Section 2.2, Küchler and Sørensen (1997): with the notations from Notation A.1.1, for a fixed $t \in(0, \infty)$, if $\boldsymbol{\theta} \mapsto C_{t}(\boldsymbol{\theta})$ is a differentiable convex map such that for any $\boldsymbol{\theta}_{0} \in$ int $\boldsymbol{\Theta}$ and $\boldsymbol{\theta}_{1} \in \boldsymbol{\Theta} \backslash$ int $\boldsymbol{\Theta}$ we have

$$
\frac{\partial}{\partial \alpha} C_{t}\left((1-\alpha) \boldsymbol{\theta}_{\mathbf{0}}+\alpha \boldsymbol{\theta}_{\mathbf{1}}\right) \longrightarrow \infty, \quad \text { as } \alpha \rightarrow 1,
$$

then $C_{t}$ is said to be steep. Note that a sufficient condition for this property to be true is to have $\Theta=\mathbb{R}^{k}$ (Küchler and Sørensen 1997, Section 2.2). In this context, we define

$$
C_{t}(\boldsymbol{\theta}):=\frac{1}{2} \int_{0}^{t}\left\langle\mathbf{Q}(\boldsymbol{\theta}) \mathbb{Y}_{s} ; \mathbf{Q}(\boldsymbol{\theta}) \mathbb{Y}_{s}\right\rangle_{\boldsymbol{\Sigma}} d s,
$$

and, from Notation 3.3.1, recall that we have

$$
\mathbb{H}_{t}:=-\left(\begin{array}{c}
\int_{0}^{t}\left\langle\overline{\mathbf{A}} \mathbb{Y}_{s}, d \mathbb{Y}_{s}^{c}\right\rangle_{\Sigma} \\
\int_{0}^{t}\left\langle\mathbb{Y}_{s}, d \mathbb{Y}_{s}^{c}\right\rangle_{\Sigma}
\end{array}\right) \text { such that }[\mathbb{H}]_{t}=\left(\begin{array}{cc}
\int_{0}^{t}\left\langle\overline{\mathbf{A}} \mathbb{Y}_{s}, \overline{\mathbf{A}} \mathbb{Y}_{s}\right\rangle_{\Sigma} d s & \int_{0}^{t}\left\langle\overline{\mathbf{A}} \mathbb{Y}_{s}, \mathbb{Y}_{s}\right\rangle_{\Sigma} d s \\
\int_{0}^{t}\left\langle\overline{\mathbf{A}} \mathbb{Y}_{s}, \mathbb{Y}_{s}\right\rangle_{\Sigma} d s & \int_{0}^{t}\left\langle\mathbb{Y}_{s}, \mathbb{Y}_{s}\right\rangle_{\Sigma} d s
\end{array}\right),
$$


and that $2 C_{t}(\boldsymbol{\theta})=\boldsymbol{\theta}^{\top} \cdot[\mathbb{H}]_{t} \cdot \boldsymbol{\theta}$ by Eq. (7).

Proof of Theorem 3.3.4 According to Proposition 3.3.3, the likelihood is defined for any $\boldsymbol{\theta} \in$ $\mathbb{R}^{2}$ and note that $C_{t}(\boldsymbol{\theta})$ is the cumulant generating function of $\mathbb{H}_{t}$. Theorem 8.2.1, Küchler and Sørensen (1997) requires that (a) the said likelihood representation is minimal which is true since $\mathbb{H}$ has almost surely affinely independent components (Küchler and Sørensen 1997, Section 4); (b) $\boldsymbol{\theta} \mapsto \boldsymbol{\theta}$ is injective which is trivially true; (c) the cumulant function $\boldsymbol{\theta} \mapsto C_{t}(\boldsymbol{\theta})$ to be defined on a set $\boldsymbol{\Theta}$ independent of $t$ and to be steep - again, this is also true since by Lemma 3.3.2 $\boldsymbol{\theta} \mapsto C_{t}(\boldsymbol{\theta})$ is defined on $\mathbb{R}^{2}$ with $|[\mathbb{H}]|_{t}<\infty$ a.s. componentwise for a fixed $t \geq 0$ large enough (Küchler and Sørensen 1997, criterion in Section 2.2).

In closed-form, we obtain from Proposition 3.3.3 and Notation A.1.1 that:

$$
\begin{aligned}
\nabla \ln \mathcal{L}_{t}(\boldsymbol{\theta} ; \mathbb{Y}) & =\left(\begin{array}{c}
-\int_{0}^{t}\left\langle\overline{\mathbf{A}} \mathbb{Y}_{s}, d \mathbb{Y}_{s}^{c}\right\rangle_{\boldsymbol{\Sigma}}-\theta_{1} \int_{0}^{t}\left\langle\overline{\mathbf{A}} \mathbb{Y}_{s}, \overline{\mathbf{A}} \mathbb{Y}_{s}\right\rangle_{\Sigma} d s-\theta_{2} \int_{0}^{t}\left\langle\overline{\mathbf{A}} \mathbb{Y}_{s}, \mathbb{Y}_{s}\right\rangle_{\boldsymbol{\Sigma}} d s \\
-\int_{0}^{t}\left\langle\mathbb{Y}_{s}, d \mathbb{Y}_{s}^{c}\right\rangle_{\boldsymbol{\Sigma}}-\theta_{1} \int_{0}^{t}\left\langle\overline{\mathbf{A}} \mathbb{Y}_{s}, \mathbb{Y}_{s}\right\rangle_{\Sigma} d s-\theta_{2} \int_{0}^{t}\left\langle\mathbb{Y}_{s}, \mathbb{Y}_{s}\right\rangle_{\Sigma} d s
\end{array}\right), \\
& =\mathbb{H}_{t}-[\mathbb{H}]_{t} \cdot \boldsymbol{\theta} .
\end{aligned}
$$

Therefore, under necessary conditions, the MLE $\widehat{\boldsymbol{\theta}}_{t}$ up to time $t \geq 0$ large enough is given by $[\mathbb{H}]_{t}^{-1} \cdot \mathbb{H}_{t}$, i.e.

$$
\widehat{\boldsymbol{\theta}}_{t}=\operatorname{det}\left([\mathbb{H}]_{t}\right)^{-1}\left(\begin{array}{c}
\int_{0}^{t}\left\langle\mathbb{Y}_{u-}, d \mathbb{Y}_{u}^{c}\right\rangle_{\Sigma} \int_{0}^{t}\left\langle\overline{\mathbf{A}} \mathbb{Y}_{v}, \mathbb{Y}_{v}\right\rangle_{\Sigma} d v-\int_{0}^{t}\left\langle\overline{\mathbf{A}} \mathbb{Y}_{u-}, d \mathbb{Y}_{u}^{c}\right\rangle_{\Sigma} \int_{0}^{t}\left\langle\mathbb{Y}_{v}, \mathbb{Y}_{v}\right\rangle_{\Sigma} d v \\
\int_{0}^{t}\left\langle\overline{\mathbf{A}} \mathbb{Y}_{u-}, d \mathbb{Y}_{u}^{c}\right\rangle_{\boldsymbol{\Sigma}} \int_{0}^{t}\left\langle\overline{\mathbf{A}} \mathbb{Y}_{v}, \mathbb{Y}_{v}\right\rangle_{\Sigma} d v-\int_{0}^{t}\left\langle\mathbb{Y}_{u-}, d \mathbb{Y}_{u}^{c}\right\rangle_{\Sigma} \int_{0}^{t}\left\langle\overline{\mathbf{A}} \mathbb{Y}_{v}, \overline{\mathbf{A}} \mathbb{Y}_{v}\right\rangle_{\Sigma} d v
\end{array}\right)
$$

Regarding the existence and uniqueness of the estimator, by application of Theorem 8.2.1, Küchler and Sørensen (1997), the maximum likelihood estimator exists and is unique if and

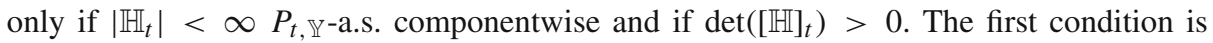
satisfied again by Lemma 3.3.2 whilst the second remains to be checked.

More precisely, the almost-sure positiveness of the determinant of the matrix $[\mathbb{H}]_{t}$ is proved using Fubini's theorem along with the Cauchy-Schwarz's inequality (in both its inner product and integral forms) as follows:

$$
\int_{0}^{t}\left\langle\overline{\mathbf{A}} \mathbb{Y}_{u}, \overline{\mathbf{A}} \mathbb{Y}_{u}\right\rangle_{\Sigma} d u \times \int_{0}^{t}\left\langle\mathbb{Y}_{v}, \mathbb{Y}_{v}\right\rangle_{\Sigma} d v \geq\left(\int_{0}^{t}\left|\left\langle\overline{\mathbf{A}} \mathbb{Y}_{u}, \mathbb{Y}_{u}\right\rangle_{\Sigma}\right| d u\right)^{2} \quad \text { since } 1 \geq \mathbb{I}\{u=v\} .
$$

Here, (ineq)equalities are almost sure with respect to the martingale measure $P_{t, \mathbb{Y}}$. Finally, observe that the Cauchy-Schwarz inequalities are actually sharp since the diagonal of $\overline{\mathbf{A}}$ is zero. More precisely, the $i$-th component of $\overline{\mathbf{A}} \mathbb{Y}_{u}$ is not linearly dependent with the $i$-th component of $\mathbb{Y}_{u}$ since diag $(\overline{\mathbf{A}})=\mathbf{0}$. Thus, we conclude since the determinant of $[\mathbb{H}]_{t}$, denoted $\operatorname{det}\left([\mathbb{H}]_{t}\right)$, has the following value:

$\operatorname{det}\left([\mathbb{H}]_{t}\right)=\int_{0}^{t}\left\langle\overline{\mathbf{A}} \mathbb{Y}_{s}, \overline{\mathbf{A}} \mathbb{Y}_{s}\right\rangle_{\Sigma} d s \times \int_{0}^{t}\left\langle\mathbb{Y}_{s}, \mathbb{Y}_{s}\right\rangle_{\Sigma} d s-\left(\int_{0}^{t}\left\langle\overline{\mathbf{A}} \mathbb{Y}_{s}, \mathbb{Y}_{s}\right\rangle_{\Sigma} d s\right)^{2}>0 \quad P_{t, \mathbb{Y}}-$ a.s.,

for any $t \geq 0$. We have then proved that this estimator exists and is unique on $\mathbb{R}^{2}$. By convexity of the likelihood, it is also unique on any compact set on which it exists which concludes the proof.

\section{A.3 Proof of Lemma 3.4.1}

Proof To prove that $\lim \inf _{t \rightarrow \infty} t^{-1} \lambda_{\min }\left(\mathbf{K}_{t}\right)>0$, one mainly uses Proposition 3.1.1 with the vectorised matrix $\mathbb{Y}_{s} \mathbb{Y}_{s}^{\top}$, denoted $\operatorname{vec}\left(\mathbb{Y}_{s} \mathbb{Y}_{s}^{\top}\right)$ which yields

$$
\frac{1}{t} \int_{0}^{t} \operatorname{vec}\left(\mathbb{Y}_{s} \mathbb{Y}_{s}^{\top}\right) d s \stackrel{\mathrm{P}_{\mathbf{0}}-a . s .}{\longrightarrow} \mathrm{E}_{t, \mathbb{Y}}\left(\operatorname{vec}\left(\mathbb{Y}_{\infty} \mathbb{Y}_{\infty}^{\top}\right)\right)<\infty, \quad \text { as } t \rightarrow \infty,
$$


which can clearly be written in its matrix form. Similarly to Masuda (2004) and Gaïffas and Matulewicz (2019), the stationary solution presented in Eq. (4) gives away that the limiting (ergodic) quantity is positive definite a.s. since we have

$$
\mathrm{E}_{t, \mathbb{Y}}\left(\mathbb{Y}_{\infty} \mathbb{Y}_{\infty}^{\top}\right)=\int_{0}^{\infty} e^{-s \mathbf{Q}} \mathrm{E}\left(\mathbb{L}_{1} \mathbb{L}_{1}^{\top}\right) e^{-s \mathbf{Q}^{\top}} d s,
$$

which is positive definite. The result follows immediately and by extension, for $t$ large enough, we can deduce that $\mathbf{K}_{t}$ is almost-surely positive definite.

\section{A.4 Proof of Proposition 3.4.2}

Proof of Proposition 3.4.2 Recall that Proposition 2.4.3 holds and we write $\widetilde{\mathbf{Q}}:=\operatorname{vec}^{-1}(\boldsymbol{\psi})$. The logarithm of the likelihood defined Eq. (6) evaluated at $\widetilde{\mathbf{Q}}$ is written as

$$
-\int_{0}^{t}\left\langle\widetilde{\mathbf{Q}} \mathbb{Y}_{s}, d \mathbb{Y}_{s}^{c}\right\rangle_{\Sigma}-\frac{1}{2} \int_{0}^{t}\left\langle\widetilde{\mathbf{Q}} \mathbb{Y}_{s}, \widetilde{\mathbf{Q}} \mathbb{Y}_{s}\right\rangle_{\Sigma} d s
$$

The first term (up to its sign) is given by $\int_{0}^{t}\left\langle\widetilde{\mathbf{Q}} \mathbb{Y}_{s}, d \mathbb{Y}_{s}^{c}\right\rangle_{\Sigma}$ which can be reformulated as follows

$$
\begin{aligned}
\int_{0}^{t}\left\langle\widetilde{\mathbf{Q}}_{s}, d \mathbb{Y}_{s}^{c}\right\rangle_{\Sigma} & =\int_{0}^{t} \sum_{n=1}^{d} \sum_{m=1}^{d} \sum_{l=1}^{d} \widetilde{Q}_{m l} Y_{s}^{(l)}\left(\Sigma^{-1}\right)_{m n} d Y_{s}^{(n), c} \\
& =\sum_{l=1}^{d} \sum_{m=1}^{d} \sum_{n=1}^{d} \widetilde{Q}_{m l}\left(\boldsymbol{\Sigma}^{-1}\right)_{m n} \int_{0}^{t} Y_{s}^{(l)} d Y_{s}^{(n), c} \\
& =\sum_{l=1}^{d} \sum_{m=1}^{d} \widetilde{Q}_{m l} \sum_{n=1}^{d}\left(\left(\boldsymbol{\Sigma}^{-1}\right)_{m n} \int_{0}^{t} Y_{s}^{(l)} d Y_{s}^{(n), c}\right) \\
& =\boldsymbol{\psi}^{\top} \cdot \mathbf{I}_{d \times d} \otimes \boldsymbol{\Sigma}^{-1} \cdot \int_{0}^{t} \mathbb{Y}_{s} \otimes d \mathbb{Y}_{s}^{c},
\end{aligned}
$$

For the second term, one obtains that

$$
\begin{aligned}
\int_{0}^{t}\left\langle\widetilde{\mathbf{Q}} \mathbb{Y}_{s}, \widetilde{\mathbf{Q}} \mathbb{Y}_{s}\right\rangle_{\Sigma} & =\int_{0}^{t} \sum_{n=1}^{d} \sum_{m=1}^{d} \sum_{l=1}^{d} \sum_{j=1}^{d} \widetilde{Q}_{m l}\left(\Sigma^{-1}\right)_{m n} Y_{s}^{(l)} Y_{s}^{(j)} \widetilde{Q}_{n j} d s \\
& =\sum_{n=1}^{d} \sum_{m=1}^{d} \sum_{l=1}^{d} \sum_{j=1}^{d} \widetilde{Q}_{m l} \int_{0}^{t} Y_{s}^{(l)} Y_{s}^{(j)} d s\left(\boldsymbol{\Sigma}^{-1}\right)_{m n} \widetilde{Q}_{n j} \\
& =\sum_{n=1}^{d} \sum_{m=1}^{d} \sum_{l=1}^{d} \sum_{j=1}^{d} \boldsymbol{\psi}_{d(l-1)+m} \int_{0}^{t} Y_{s}^{(l)} Y_{s}^{(j)} d s\left(\boldsymbol{\Sigma}^{-1}\right)_{m n} \boldsymbol{\psi}_{d(j-1)+n}
\end{aligned}
$$

Since $\psi=\operatorname{vec}(\widetilde{\mathbf{Q}})$, we have that

$$
\int_{0}^{t}\left\langle\widetilde{\mathbf{Q}} \mathbb{Y}_{s}, \widetilde{\mathbf{Q}} \mathbb{Y}_{s}\right\rangle_{\Sigma} d s=\boldsymbol{\psi}^{\top} \cdot \int_{0}^{t} \mathbb{Y}_{s} \mathbb{Y}_{s}^{\top} d s \otimes \Sigma^{-1} \cdot \boldsymbol{\psi}
$$


Finally, using that $(A C) \otimes(B D)=(A \otimes B) \cdot(C \otimes D)$ with $A, B, C, D$ four matrices with dimensions such that the products $A C$ and $B D$ are well-defined, one obtains

$$
\int_{0}^{t}\left\langle\widetilde{\mathbf{Q}} \mathbb{Y}_{s}, \widetilde{\mathbf{Q}} \mathbb{Y}_{s}\right\rangle_{\Sigma} d s=\boldsymbol{\psi}^{\top} \cdot\left(\mathbf{I}_{d \times d} \otimes \Sigma^{-1}\right) \cdot\left(\mathbf{K}_{t} \otimes \mathbf{I}_{d \times d}\right) \cdot \boldsymbol{\psi}=\boldsymbol{\psi}^{\top} \cdot[\mathbb{I}]_{t} \cdot \boldsymbol{\psi},
$$

which concludes the proof. Given the stationary and square integrability of $\mathbb{Y}, \mathbb{I}$ and $[\mathbb{I}]$ are a.s. finite for $t<\infty$ and we have that $\boldsymbol{\Psi}:=\left\{\boldsymbol{\psi} \in \mathbb{R}^{d^{2}}:\left|\boldsymbol{\psi}^{\top} \cdot[\mathbb{I}]_{t} \cdot \boldsymbol{\psi}\right|<\infty, \forall t \geq 0\right\}=\mathbb{R}^{d^{2}}$.

\section{A.5 Proof of Proposition 4.1.1}

Proof of Proposition 4.1.1 Similarly to Sect. A.2, we define

$$
C_{t}(\boldsymbol{\theta}):=\frac{1}{2} \int_{0}^{t}\left\langle\mathbf{Q}(\boldsymbol{\theta}) \mathbb{Y}_{s} ; \mathbf{Q}(\boldsymbol{\theta}) \mathbb{Y}_{s}\right\rangle_{\Sigma} d s .
$$

We verify point-by-point the conditions of Theorem 8.3.1, Küchler and Sørensen (1997) to show the consistency of the MLE under $P_{\mathbb{Y}}$. Let $\boldsymbol{\theta} \in \widehat{\boldsymbol{\Theta}}$.

First, Lemma 3.3.2, we have

$$
t^{-1}[\mathbb{H}]_{t} \stackrel{\mathrm{P}_{0}-\text { a.s. }}{\longrightarrow} \boldsymbol{G}_{\infty}
$$

which is positive definite by Cauchy-Schwarz's inequality (see Sect. A.2) and independent of $\boldsymbol{\theta}$ ensuring that

$$
\left\|t^{-1}[\mathbb{H}]_{t}-\boldsymbol{G}_{\infty}\right\| \stackrel{p}{\longrightarrow} 0, \quad \text { as } t \rightarrow \infty .
$$

Then, we prove that $[\mathbb{H}]_{t}^{-1 / 2} \cdot\left(\mathbb{H}_{t}-\nabla C_{t}(\boldsymbol{\theta})\right)$ is stochastically bounded for $t \geq 0$ large enough. It requires to prove that for any $\epsilon>0$, there exists $K>0$ such that

$$
\sup _{\boldsymbol{\theta} \in \widetilde{\boldsymbol{\Theta}}} \mathrm{P}_{t, \mathbb{Y}}\left\{\left|t^{-1 / 2} \boldsymbol{G}_{\infty}^{-1 / 2} \cdot\left(\mathbb{H}_{t}-\nabla C_{t}(\boldsymbol{\theta})\right)\right|>K\right\}<\epsilon .
$$

Next, by ergodicity we have that:

$$
[\mathbb{H}]_{t}^{-1 / 2} \cdot \mathbb{H}_{t}=t^{1 / 2}\left([\mathbb{H}]_{t} / t\right)^{-1 / 2} \mathbb{H}_{t} / t=O\left(t^{1 / 2}\right), \quad \mathrm{P}_{0}-\text { a.s. }
$$

as $t \rightarrow \infty$ independently of $\boldsymbol{\theta}$. Recall that $\nabla C_{t}(\boldsymbol{\theta})=[\mathbb{H}]_{t} \cdot \boldsymbol{\theta}$. We have that $[\mathbb{H}]_{t}^{-1 / 2} \cdot \nabla C_{t}(\boldsymbol{\theta})$ has almost-surely finite components for any $t \geq 0$. Indeed, those are càdlàg quantities which converge almost surely to a quantity that is $O\left(t^{1 / 2}\right)$ as $t \rightarrow \infty$. Therefore, those components cannot explode with a probability larger than 0 for any $t \geq 0$ large enough - say above some fixed $t_{1} \geq 0$. This is valid on the compact set $\widetilde{\Theta}$ hence we have proved the uniform stochastic boundedness of $\left\{[\mathbb{H}]_{t}^{1 / 2} \cdot\left(\mathbb{H}_{t}-\nabla C_{t}(\boldsymbol{\theta})\right): t \geq t_{1}\right\}$ on $\widehat{\boldsymbol{\Theta}}$. Therefore, thanks to the latter and (24), Theorem 8.3.1, Küchler and Sørensen (1997) yields that $\widehat{\boldsymbol{\theta}_{t}} \stackrel{p}{\longrightarrow} \boldsymbol{\theta}$ as $t \rightarrow \infty$.

\section{A.6 Proof of Lemma 4.1.2}

We prove that the likelihood converges locally to a Gaussian shift experiment (Hájek 1970). Since $d \mathbb{Y}_{t}^{c}=d \mathbb{W}_{t}-\mathbf{Q}(\boldsymbol{\theta}) \mathbb{Y}_{t-} d t$, we decompose $\mathbb{H}$ into a stochastic term $\mathbb{H}^{(s)}$ and a drift 
term $\mathbb{H}^{(d)}$ defined for any $t \geq 0$ by

$$
\mathbb{H}_{t}^{(s)}:=-\left(\begin{array}{c}
\int_{0}^{t}\left\langle\overline{\mathbf{A}} \mathbb{Y}_{s}, d \mathbb{W}_{s}\right\rangle_{\boldsymbol{\Sigma}} \\
\int_{0}^{t}\left\langle\mathbb{Y}_{s}, d \mathbb{W}_{s}\right\rangle_{\boldsymbol{\Sigma}}
\end{array}\right) \text { and } \mathbb{H}_{t}^{(d)}:=\left(\begin{array}{c}
\int_{0}^{t}\left\langle\overline{\mathbf{A}} \mathbb{Y}_{s}, \mathbf{Q}(\boldsymbol{\theta}) \mathbb{Y}\right\rangle_{\boldsymbol{\Sigma}} d s \\
\int_{0}^{t}\left\langle\mathbb{Y}_{s}, \mathbf{Q}(\boldsymbol{\theta}) \mathbb{Y}_{s}\right\rangle_{\boldsymbol{\Sigma}} d s
\end{array}\right),
$$

such that $\mathbb{H}_{t} \stackrel{d}{=} \mathbb{H}_{t}^{(s)}+\mathbb{H}_{t}^{(d)}$. We note that

$$
\mathbb{H}_{t}^{(d)} \stackrel{d}{=}[\mathbb{H}]_{t} \boldsymbol{\theta},
$$

since $\mathbf{Q}(\boldsymbol{\theta})=\theta_{2} \mathbf{I}_{d \times d}+\theta_{1} \overline{\mathbf{A}}$. According to Proposition 3.3.3, we define the log-likelihood $\ell_{t}(\boldsymbol{\theta} ; \mathbb{Y}):=\ln \mathcal{L}_{t}(\boldsymbol{\theta} ; \mathbb{Y})$ for $\boldsymbol{\theta} \in \widehat{\boldsymbol{\Theta}}$. By the almost sure finiteness of $\mathbb{H}$ and $[\mathbb{H}]$, for any $\boldsymbol{h} \in \mathbb{R}^{2}$, we obtain as increment of the log-likelihood the following:

$$
\begin{aligned}
\ell_{t}\left(\boldsymbol{\theta}+t^{-1 / 2} \boldsymbol{h} ; \mathbb{Y}\right)-\ell_{t}(\boldsymbol{\theta} ; \mathbb{Y}) & =t^{-1 / 2} \boldsymbol{h}^{\top} \mathbb{H}_{t}-t^{-1 / 2} \boldsymbol{h}^{\top}[\mathbb{H}]_{t} \boldsymbol{\theta}-\frac{t^{-1}}{2} \boldsymbol{h}^{\top}[\mathbb{H}]_{t} \boldsymbol{h} \\
& =t^{-1 / 2} \boldsymbol{h}^{\top} \mathbb{H}_{t}^{(s)}+t^{-1 / 2} \boldsymbol{h}^{\top} \mathbb{H}_{t}^{(d)}-t^{-1 / 2} \boldsymbol{h}^{\top}[\mathbb{H}]_{t} \boldsymbol{\theta}-\frac{t^{-1}}{2} \boldsymbol{h}^{\top}[\mathbb{H}]_{t} \boldsymbol{h} \\
& =t^{-1 / 2} \boldsymbol{h}^{\top} \mathbb{H}_{t}^{(s)}-\frac{t^{-1}}{2} \boldsymbol{h}^{\top}[\mathbb{H}]_{t} \boldsymbol{h}, \quad \text { by Eq. (25). }
\end{aligned}
$$

Then, we observe that: (i) $\mathbb{H}^{(s)}$ is a square integrable martingale (under $\mathrm{P}_{\mathbb{Y}}^{\boldsymbol{\theta}}$ ) by the square integrability of $\mathbb{Y}$; (ii) $\mathbb{H}^{(s)}$ is continuous hence $\mathbb{H}_{t}^{(s)}-\mathbb{H}_{t-}^{(s)}=0$ because $\mathbb{Y}$ is càdlàg; (iii) $t^{-1}[\mathbb{H}]_{t} \rightarrow \boldsymbol{G}_{\infty}, \mathrm{P}_{0}$-almost surely (by Theorem 3.1.1) hence in probability where $\boldsymbol{G}_{\infty}$ is a (deterministic) positive definite matrix. This also yields the convergence of the covariance matrix of $\mathbb{H}_{t}^{(s)}$ as $t \rightarrow \infty$. Then, by Theorem A.7.7, Küchler and Sørensen (1997), we obtain the pairwise convergence

$$
\left(t^{-1 / 2} \mathbb{H}_{t}^{(s)}, \frac{t^{-1}}{2}[\mathbb{H}]_{t}\right) \stackrel{\mathcal{D}}{\longrightarrow}\left(\boldsymbol{G}_{\infty}^{1 / 2} \eta, \frac{1}{2} \boldsymbol{G}_{\infty}\right), \quad \text { as } t \rightarrow \infty,
$$

where $\boldsymbol{\eta}$ is a 2-dimensional standard normal random variable and therefore

$$
l\left(\boldsymbol{\theta}+t^{-1 / 2} \boldsymbol{h} ; \mathbb{Y}\right)-l(\boldsymbol{\theta} ; \mathbb{Y}) \stackrel{\mathcal{D}}{\rightarrow} \boldsymbol{h}^{\top} \boldsymbol{G}_{\infty}^{1 / 2} \boldsymbol{\eta}-\frac{1}{2} \boldsymbol{h}^{\top} \boldsymbol{G}_{\infty} \boldsymbol{h}, \quad \text { as } t \rightarrow \infty .
$$

We conclude that the family of statistical models $\left(\mathrm{P}_{\mathbb{Y}}^{\boldsymbol{\theta}}, \boldsymbol{\theta} \in \widehat{\boldsymbol{\Theta}}\right)$ is locally asymptotically normal since $\boldsymbol{G}_{\infty}$ is deterministic.

\section{A.7 Proof of Theorem 4.1.3}

Proof of Theorem 4.1.3 Using the notations of Sect. A.5 (proof of Proposition 4.1.1), we have that $t \mapsto \nabla C_{t}(\boldsymbol{\theta})=[\mathbb{H}]_{t} \cdot \boldsymbol{\theta}$ is continuous with bounded variation on compact intervals as a time integral of an $L^{1}$ integrand. Condition 8.3.2, Küchler and Sørensen (1997) holds since: (a) $\mathbb{H}_{t}$ is continuously-valued in time; (b) $t^{-1} \nabla^{2} C_{t}(\boldsymbol{\theta})=t^{-1}[\mathbb{H}]_{t} \rightarrow \boldsymbol{G}_{\infty}$ under $\mathrm{P}_{\mathbb{Y}}$ which is positive definite; (c) by Fubini-Tonelli's theorem, we have the same limit for the expected information matrix

$$
t^{-1} \cdot \mathrm{E}\left([\mathbb{H}]_{t}\right) \stackrel{\mathrm{P}_{0}-a . s .}{\longrightarrow} \boldsymbol{G}_{\infty},
$$

since $\mathbb{Y}_{s}$ has finite second moments for any $s \geq 0$. Hence, by Theorem 8.3.4, Küchler and Sørensen (1997), we obtain that

$$
[\mathbb{H}]_{t}^{1 / 2}\left(\widehat{\boldsymbol{\theta}}_{t}-\boldsymbol{\theta}\right) \stackrel{\mathcal{D}}{\longrightarrow} \mathcal{N}\left(\mathbf{0}, I_{2 \times 2}\right), \quad \text { as } t \rightarrow \infty .
$$


Regarding the asymptotic efficiency, recall that $\widehat{\boldsymbol{\theta}}:=[\mathbb{H}]_{t}^{-1} \mathbb{H}_{t}$ and from Lemma 4.1.2 and its proof (Eqs. (25) and (26)), we obtain that that $t^{1 / 2}(\widehat{\boldsymbol{\theta}}-\boldsymbol{\theta})$ is asymptotically Gaussian with an asymptotic variance of $\boldsymbol{G}_{\infty}$ which is the corresponding Fisher information matrix. According to the Hájek-Le Cam's convolution theorem for locally asymptotically normal experiments (Le Cam and Lo Yang 1990), we obtain the Hájek-Le Cam asymptotic efficiency of the estimator.

\section{A.8 Proof of Lemma 4.2.1}

Proof of Lemma 4.2.1 Similarly to Example 8.3.6, Küchler and Sørensen (1997), we notice that Eq. (1) in particular for the continuous part of $\mathbb{Y}$ yields $d \mathbb{Y}_{t}^{c}=-\mathbf{Q} \mathbb{Y}_{t} d t+d \mathbb{W}_{t}$. Since $\widehat{\boldsymbol{\psi}}$ can be written $[\mathbb{I}]_{t}^{-1} \mathbb{I}_{t}$, we have $\widehat{\boldsymbol{\psi}}_{t}=[\mathbb{I}]_{t}^{-1}\left([\mathbb{I}]_{t} \boldsymbol{\psi}+\mathbb{M}_{t}\right)=\boldsymbol{\psi}+[\mathbb{I}]_{t}^{-1} \mathbb{M}_{t}$ where $\mathbb{M}_{t}=\int_{0}^{t} \mathbb{Y}_{t} \otimes d \mathbb{W}_{t}$. In addition, $\mathbb{M}_{t}$ is a martingale under $\mathrm{P}_{0}$ since $\mathbb{Y}_{t} \in L^{2}\left(\mathbb{R}^{d}\right)$.

\section{A.9 Proof of Theorem 4.2.3}

Proof of Theorem 4.2.3 Denote $G_{\infty}^{\psi}:=\mathrm{E}\left(\mathbb{Y}_{\infty} \mathbb{Y}_{\infty}^{\top}\right) \otimes \Sigma^{-1}$. The proof is similar to that of Prop. 4.1.1 and we define $\widetilde{\mathbf{Q}}:=\operatorname{vec}^{-1}(\boldsymbol{\psi})$ as well as

$$
C_{t}(\boldsymbol{\psi}):=\frac{1}{2} \int_{0}^{t}\left\langle\widetilde{\mathbf{Q}} \mathbb{Y}_{s}, \widetilde{\mathbf{Q}} \mathbb{Y}_{s}\right\rangle_{\Sigma} d s=\frac{1}{2} \boldsymbol{\psi}^{\top} \cdot[\mathbb{I}]_{t} \cdot \boldsymbol{\psi} .
$$

Observe that $\nabla^{2} C_{t}(\boldsymbol{\psi})=[\mathbb{I}]_{t}=\mathbf{K}_{t} \otimes \boldsymbol{\Sigma}^{-1}$ and we have by ergodicity

$$
t^{-1}[\mathbb{I}]_{t} \longrightarrow \mathrm{E}\left(\mathbb{Y}_{\infty} \mathbb{Y}_{\infty}^{\top}\right) \otimes \Sigma^{-1}=G_{\infty}^{\psi} \quad \mathrm{P}_{0}-\text { a.s. }, \quad \text { as } t \rightarrow \infty
$$

since $t^{-1} \int_{0}^{t} Y_{s}^{(i)} Y_{s}^{(j)} d s \rightarrow \mathrm{E}\left(Y_{\infty}^{(i)} Y_{\infty}^{(j)}\right) \mathrm{P}_{0}-$ a.s. as $t \rightarrow \infty$. Note that $t^{-1} \mathrm{E}\left([\mathbb{I}]_{t}\right)$ converges to the same limit and that

$$
[\mathbb{I}]_{t}^{-1 / 2} \mathbb{I}_{t}=t^{1 / 2} \cdot\left([\mathbb{I}]_{t} / t\right)^{-1 / 2} \cdot \mathbb{I} / t=O\left(t^{1 / 2}\right) \quad \mathrm{P}_{0}-\text { a.s., as } t \rightarrow \infty,
$$

since $\boldsymbol{G}_{\infty}^{\psi}$ has almost-surely finite components and positive definite.

Theorem 8.3.4, Küchler and Sørensen (1997) gives a central limit theorem with $[\mathbb{I}]_{t}^{1 / 2}$ as the scaling matrix. Since the stochastic boundedness and finite variation over bounded intervals of $\left\{[\mathbb{I}]_{t}^{-1}\left(\mathbb{I}_{t}-\nabla C_{t}(\boldsymbol{\psi})\right): t \geq 0\right\}$ on $\widehat{\boldsymbol{\Psi}}$ can be proved similarly to the proof of Proposition 4.1.1, we can now apply Theorem 8.3.4, Küchler and Sørensen (1997) and conclude that, as $t \rightarrow \infty$,

$$
[\mathbb{I}]_{t}^{-1 / 2}\left(\widehat{\boldsymbol{\psi}}_{t}-\boldsymbol{\psi}\right) \stackrel{\mathcal{D}}{\longrightarrow} \mathcal{N}\left(\mathbf{0}_{d^{2}}, \mathbf{I}_{d^{2} \times d^{2}}\right) .
$$

and, by Slutsky's lemma,

$$
t^{1 / 2}\left(\widehat{\boldsymbol{\psi}}_{t}-\boldsymbol{\psi}\right) \stackrel{\mathcal{D}}{\longrightarrow} \mathcal{N}\left(\mathbf{0}_{d^{2}}, \mathrm{E}\left(\mathbb{Y}_{\infty} \mathbb{Y}_{\infty}^{\top}\right)^{-1} \otimes \boldsymbol{\Sigma}\right)
$$




\section{A.10 Proof of Theorem 5.3.1}

Proof We first show the asymptotic normality property before leveraging it to prove the consistency in variable selection of the Adaptive Lasso. Since $d \mathbb{Y}_{t}^{c}=-\mathbf{Q}_{0} \mathbb{Y}_{t-} d t+d \mathbb{W}_{t}$, we center the log-likelihood around $\mathbf{Q}_{0}$ as follows:

$\ell_{t}(\mathbf{Q})=-\int_{0}^{t}\left\langle\mathbf{Q} \mathbb{Y}_{s}, d \mathbb{W}_{s}\right\rangle_{\Sigma}-\frac{1}{2} \int_{0}^{t}\left\langle\left(\mathbf{Q}-\mathbf{Q}_{0}\right) \mathbb{Y}_{s},\left(\mathbf{Q}-\mathbf{Q}_{0}\right) \mathbb{Y}_{s}\right\rangle_{\Sigma} d s+\frac{1}{2} \int_{0}^{t}\left\langle\mathbf{Q}_{0} \mathbb{Y}_{s}, \mathbf{Q}_{0} \mathbb{Y}_{s}\right\rangle_{\Sigma} d s$.

such that $\ell_{t}(\mathbf{Q})-\ell_{t}\left(\mathbf{Q}_{0}\right)=-\int_{0}^{t}\left\langle\left(\mathbf{Q}-\mathbf{Q}_{0}\right) \mathbb{Y}_{s}, d \mathbb{W}_{s}\right\rangle_{\Sigma}-\frac{1}{2} \int_{0}^{t}\left\langle\left(\mathbf{Q}-\mathbf{Q}_{0}\right) \mathbb{Y}_{s},\left(\mathbf{Q}-\mathbf{Q}_{0}\right) \mathbb{Y}_{s}\right\rangle_{\Sigma} d s$. Without loss of generality, we change the penalty rate from $\lambda$ to $\lambda t$ since it does not depend on $\mathbf{Q}$. Then, by writing $\mathbf{Q}=\mathbf{Q}_{0}+t^{-1 / 2} \mathbf{M}$ for some $\mathbf{M} \in \mathcal{M}_{d}(\mathbb{R})$, we have

$$
t^{1 / 2}\left(\widehat{\mathbf{Q}}_{\mathrm{AL}, t}-\mathbf{Q}_{0}\right)_{\mid \mathcal{Q}_{0}}=\underset{\mathbf{M} \in \mathcal{M}_{d}(\mathbb{R})}{\arg \max } \kappa_{t}^{1}(\mathbf{M})+\kappa_{t}^{2}(\mathbf{M}),
$$

where

$$
\left\{\begin{array}{l}
\kappa_{t}^{1}(\mathbf{M})=-t^{-1 / 2} \int_{0}^{t}\left\langle\mathbf{M} \mathbb{Y}_{s-}, d \mathbb{W}_{s}\right\rangle_{\boldsymbol{\Sigma}}-\frac{t^{-1}}{2} \int_{0}^{t}\left\langle\mathbf{M} \mathbb{Y}_{s}, \mathbf{M} \mathbb{Y}_{s}\right\rangle_{\boldsymbol{\Sigma}} d s \\
\kappa_{t}^{2}(\mathbf{M})=\lambda t\left\|\mathbf{Q}_{0} \odot\left|\widehat{\mathbf{Q}}_{t}\right|^{-\gamma}\right\|_{1}-\lambda t\left\|\left(\mathbf{Q}_{0}+t^{-1 / 2} \mathbf{M}\right) \odot\left|\widehat{\mathbf{Q}}_{t}\right|^{-\gamma}\right\|_{1} .
\end{array}\right.
$$

For the first function $\kappa_{t}^{1}$ We define the process $\mathrm{M}_{t}:=\int_{0}^{t}\left\langle\mathbf{M} \mathbb{Y}_{s-}, d \mathbb{W}_{s}\right\rangle_{\Sigma} d s$ and note that $[\mathrm{M}]_{t}=\int_{0}^{t}\left\langle\mathbf{M} \mathbb{Y}_{s-}, \mathbf{M} \mathbb{Y}_{s-}\right\rangle_{\Sigma} d s$ such that $\kappa_{t}^{1}(\mathbf{M})=-t^{-1 / 2} \mathbf{M}_{t}-\frac{1}{2} t^{-1}[\mathrm{M}]_{t}$. We proceed similarly to the proof of Lemma 4.1.2 (Appendix A.6): (i) $\mathrm{M}_{t}$ is a square integrable martingale (under $\mathrm{P}_{\mathbb{Y}}$ ) by the square integrability of $\mathbb{Y}$; (ii) $\mathrm{M}_{t}$ is continuous hence $\mathrm{M}_{t}-\mathrm{M}_{t-}=0$ because $\mathbb{Y}$ is càdlàg; (iii) we have the following ergodic convergence (Prop. 3.1.1) as $t \rightarrow \infty$ :

$$
t^{-1} \int_{0}^{t}\left\langle\mathbf{M} \mathbb{Y}_{s}, \mathbf{M} \mathbb{Y}_{s}\right\rangle_{\Sigma} d s \rightarrow \mathrm{E}\left(\left\langle\mathbf{M} \mathbb{Y}_{\infty}, \mathbf{M} \mathbb{Y}_{\infty}\right\rangle_{\Sigma}\right), \mathrm{P}_{\mathbf{0}}-\text { a.s. }
$$

and we obtain the convergence in probability, too. Similarly to the proof of Prop. 3.4.2 (App. A.4), namely Eq. (23), we have

$$
\mathrm{E}\left(\left\langle\mathbf{M} \mathbb{Y}_{\infty}, \mathbf{M} \mathbb{Y}_{\infty}\right\rangle_{\boldsymbol{\Sigma}}\right)=\operatorname{vec}(\mathbf{M})^{\top} \cdot \mathbf{K}_{\infty} \otimes \Sigma^{-1} \cdot \operatorname{vec}(\mathbf{M})=: K_{\infty}^{1}
$$

where $\mathbf{K}_{\infty}:=E\left(\mathbb{Y}_{\infty} \mathbb{Y}_{\infty}^{\top}\right)$. From those conditions, Th. A.7.7, Küchler and Sørensen (1997) yields the pairwise convergence

$$
\left(t^{-1 / 2} \mathrm{M}_{t}, \frac{t^{-1}}{2}[\mathrm{M}]_{t}\right) \stackrel{\mathcal{D}}{\longrightarrow}\left(\left(K_{\infty}^{1}\right)^{1 / 2} Z, \frac{K_{\infty}^{1}}{2}\right), \quad \text { as } t \rightarrow \infty,
$$

where $Z$ is a standard Gaussian random variable on the same probability space as $\mathrm{M}_{t}$. Hence, since $K_{\infty}^{1}$ is deterministic and positive, we obtain $t^{-1 / 2} \mathrm{M}_{t} \stackrel{\mathcal{D}}{\longrightarrow}\left(K_{\infty}^{1}\right)^{1 / 2} Z$ as $t \rightarrow \infty$. A distribution trick (Gaïfas and Matulewicz 2019, Section 6.6) uses a $d \times d$ Gaussian matrix $\boldsymbol{Z}$ with mean zero and covariance $\operatorname{Cov}(\operatorname{vec}(\boldsymbol{Z})$, $\operatorname{vec}(\boldsymbol{Z}))=\boldsymbol{K}_{\infty} \otimes \boldsymbol{\Sigma}^{-1}$, that is, $\operatorname{Cov}\left(\boldsymbol{Z}_{i j}, \boldsymbol{Z}_{k l}\right)=\left(\boldsymbol{K}_{\infty}\right)_{i k} \cdot \boldsymbol{\Sigma}_{j l}^{-1}$. Then, for any $\mathbf{M} \in \mathcal{M}_{d}(\mathbb{R}), \operatorname{tr}(\mathbf{M} \boldsymbol{Z})$ is a Gaussian random variable with mean zero and variance

$$
\operatorname{Var}(\operatorname{tr}(\mathbf{M} Z))=\sum_{i j k l} \mathbf{M}_{j i} \mathbf{M}_{l k} \operatorname{Cov}\left(\boldsymbol{Z}_{i j}, \boldsymbol{Z}_{k l}\right)=\sum_{i j k l} \mathbf{M}_{j i} \cdot\left(\boldsymbol{K}_{\infty}\right)_{i k} \cdot \boldsymbol{\Sigma}_{j l}^{-1} \cdot \mathbf{M}_{l k}=K_{\infty}^{1} .
$$

Using this reformulation and (27), we have

$$
\kappa_{t}^{1}(\mathbf{M}) \stackrel{\mathcal{D}}{\longrightarrow}-K_{\infty}^{1} / 2-\operatorname{tr}(\mathbf{M} Z), \quad \text { as } t \rightarrow \infty .
$$


For the second function $\kappa_{t}^{2}$ Explicitly, we have

$$
\kappa_{t}^{2}(\mathbf{M})=-\lambda t \sum_{i j}\left|\widehat{\mathbf{Q}}_{t, i j}\right|^{-\gamma}\left(\left|\mathbf{Q}_{0, i j}+t^{-1 / 2} \mathbf{M}_{i j}\right|-\left|\mathbf{Q}_{0, i j}\right|\right) .
$$

For $(i, j) \in \mathcal{Q}_{0}$, we have $\left.t^{1 / 2}\right|^{-\gamma}\left(\left|\mathbf{Q}_{0, i j}+t^{-1 / 2} \mathbf{M}_{i j}\right|-\left|\mathbf{Q}_{0, i j}\right|\right) \rightarrow \operatorname{sign}\left(\mathbf{Q}_{0, i j}\right)\left|\mathbf{M}_{i j}\right|$ as $t \rightarrow \infty$, where $\operatorname{sign}(x):=\mathbb{I}_{\{z \geq 0\}}-\mathbb{I}_{\{z<0\}}$. By the consistency of the MLE and the continuous mapping theorem, we have $\left|\widehat{\mathbf{Q}}_{t, i j}\right|^{-\gamma} \stackrel{p}{\longrightarrow}\left|\mathbf{Q}_{0, i j}\right|^{-\gamma}>0$. By assumption $\lambda t^{1 / 2}=\lambda(t) t^{1 / 2} \rightarrow$ 0 and therefore we have

$$
\lambda(t) t^{1 / 2}\left|\widehat{\mathbf{Q}}_{t, i j}\right|^{-\gamma}\left(\left|\mathbf{Q}_{0, i j}+t^{-1 / 2} \mathbf{M}_{i j}\right|-\left|\mathbf{Q}_{0, i j}\right|\right) \stackrel{p}{\longrightarrow} 0, \quad \text { as } t \rightarrow \infty .
$$

Otherwise, for $(i, j) \in \overline{\mathcal{Q}}_{0}:=\left\{(k, l) \notin \mathcal{Q}_{0}: 1 \leq k, l \leq d\right\}$, we proceed similarly but, from $t^{1 / 2}$-consistency of the MLE, we have $\left|t^{1 / 2} \widehat{\mathbf{Q}}_{t, i j}\right|=o_{p}(1)$. Hence, since $\lambda(t) t^{(\gamma+1) / 2} \rightarrow \infty$ and $\gamma>0$, we obtain that

$$
-\lambda(t) t^{(\gamma+1) / 2}\left|t^{1 / 2} \widehat{\mathbf{Q}}_{t, i j}\right|^{-\gamma}\left|\mathbf{M}_{i j}\right| \stackrel{p}{\longrightarrow}-\infty, \quad \text { if } \mathbf{M}_{i j} \neq 0, \text { as } t \rightarrow \infty .
$$

and this quantity is zero if $\mathbf{M}_{i j}=0$.

We summarise the resulting asymptotic properties as follows:

$$
\kappa_{t}^{1}(\mathbf{M})+\kappa_{t}^{2}(\mathbf{M}) \stackrel{\mathcal{D}}{\longrightarrow} \begin{cases}-\infty, & \text { if } \operatorname{supp}(\mathbf{M}) \cap \overline{\mathcal{G}_{0}} \neq \emptyset \\ -K_{\infty}^{1} / 2-\operatorname{tr}(\mathbf{M Z}), & \text { otherwise }\end{cases}
$$

Suppose that the objective function is well-defined, i.e. $\operatorname{supp}(\mathbf{M}) \cap \overline{\mathcal{G}_{0}}=\varnothing$ : the entries from $\mathbf{M}$ with indices outside the support of $\boldsymbol{Q}_{0}$ are all zero. In that case, we have $\operatorname{tr}(\mathbf{M Z})=$ $\operatorname{vec}\left(\mathbf{M}_{\mid \mathcal{G}_{0}}\right)^{\top} \cdot \operatorname{vec}\left(\boldsymbol{Z}_{\mid \mathcal{G}_{0}}^{\top}\right)$. Also, we have $K_{\infty}^{1}=\operatorname{vec}\left(\mathbf{M}_{\mid \mathcal{G}_{0}}\right)^{\top} \cdot\left(\mathbf{K}_{\infty} \otimes \boldsymbol{\Sigma}^{-1}\right)_{\mid \mathcal{G}_{0} \times \mathcal{G}_{0}} \cdot \operatorname{vec}\left(\mathbf{M}_{\mid \mathcal{G}_{0}}\right)$ and we conclude that the objective function is quadratic in $\operatorname{vec}\left(\mathbf{M}_{\mid \mathcal{G}_{0}}\right)$. Its maximum in the limit $t \rightarrow \infty$, denoted $\widehat{\mathbf{M}}$, is given by

$$
\operatorname{vec}\left(\widehat{\mathbf{M}}_{\mid \mathcal{G}_{0}}\right):=-\left(\mathbf{K}_{\infty}^{-1} \otimes \boldsymbol{\Sigma}\right)_{\mid \mathcal{G}_{0} \times \mathcal{G}_{0}} \cdot \operatorname{vec}\left(\boldsymbol{Z}_{\mid \mathcal{G}_{0}}^{\top}\right), \text { and } \widehat{\mathbf{M}}_{\mid \overline{\mathcal{G}_{0}}}:=0
$$

Given the covariance structure of $\boldsymbol{Z}$, vec $\left(\widehat{\mathbf{M}}_{\mid \mathcal{G}_{0}}\right)$ is a centred Gaussian random vector with covariance $\left(\mathbf{K}_{\infty}^{-1} \otimes \boldsymbol{\Sigma}\right)_{\mid \mathcal{G}_{0} \times \mathcal{G}_{0}}$ which concludes the proof of asymptotic normality.

Consistency in variable selection We first note that the asymptotic normality of $\widehat{\mathbf{Q}}_{\mathrm{AL}, t}$ on $\mathcal{G}_{0}$ yields that $\mathrm{P}\left(\left(\widehat{\mathbf{Q}}_{\mathrm{AL}, t}\right)_{i j} \neq 0\right) \rightarrow 1$ if $(i, j) \in \mathcal{G}_{0}$. It remains to show that $\mathrm{P}\left(\left(\widehat{\mathbf{Q}}_{\mathrm{AL}, t}\right)_{i j}=0\right) \rightarrow$ 1 if $(i, j) \in \overline{\mathcal{G}_{0}}$. Suppose that $\left(\widehat{\mathbf{Q}}_{\mathrm{AL}, t}\right)_{i j} \neq 0$. We first take the derivative of the objective function with respect to the $(i, j)$-th parameter of $\widehat{\mathbf{Q}}_{\mathrm{AL}, t}$. We multiply by $t^{-1 / 2}$, set it to zero before taking the absolute value on both sides. This yields the following relationship:

$$
\left|t^{-1 / 2} \int_{0}^{t} Y_{s}^{(j)} d W_{s}^{(i)}+\left(t^{1 / 2} \operatorname{vec}\left(\widehat{\mathbf{Q}}_{\mathrm{AL}, t}\right)^{\top} \cdot\left(t^{-1} \mathbf{K}_{t}\right) \otimes \boldsymbol{\Sigma}^{-1}\right)_{d(j-1)+i}\right|=\lambda t^{(\gamma+1) / 2} \cdot\left|t^{1 / 2} \widehat{\mathbf{Q}}_{t}\right|_{i j}^{-\gamma} .
$$

On the right hand side, as mentioned above, we have $t^{1 / 2}\left(\widehat{\mathbf{Q}}_{\mathrm{AL}, t}\right)_{\mid \overline{\mathcal{G}}_{0}}=o_{p}(1)$. Since $\lambda t^{(\gamma+1) / 2}=O(1)$ and $\gamma>0$, this side diverges to $\infty$ in probability as $t \rightarrow \infty$. The first term on the left-hand side is a martingale and is normally-distributed as $t \rightarrow \infty$. By the asymptotic normality of $t^{1 / 2}\left(\widehat{\mathbf{Q}}_{\mathrm{AL}, t}\right)_{\mid \overline{\mathcal{G}}_{0}}$ and the fact that $t^{-1} \mathbf{K}_{t} \stackrel{p}{\rightarrow} \mathbf{K}_{\infty}$, the left-hand side is the absolute value of the sum of two Gaussian random variables whose probability to be larger or equal to the right-hand side (which diverges to $\infty$ ) tends to zero. The right-hand side was computed under the assumption that $\left(\widehat{\mathbf{Q}}_{\mathrm{AL}, t}\right)_{i j} \neq 0$, and a bound of the probability of that event happening is zero, hence we have that $\mathrm{P}\left(\left(\widehat{\mathbf{Q}}_{\mathrm{AL}, t}\right)_{i j}=0\right) \rightarrow 1$ if $(i, j) \in \overline{\mathcal{G}_{0}}$. 
This shows the consistency in variable selection of the scheme, which concludes the proof.

\section{A.11 Proof of Proposition 6.3.1}

Proof of Proposition 6.3.1 We define $\pi_{V}:=\delta_{V}$ a probability measure on $\mathbb{S}_{d}^{++}$and there exists an $\mathbb{S}_{d}^{+}$-valued Lévy basis $\Lambda_{L}$ on $\mathbb{S}_{d}^{++} \times \mathbb{R}$ corresponding to the characteristic quadruplet $\left(\boldsymbol{\gamma}_{\boldsymbol{L}}, \mathbf{0}, v_{\boldsymbol{L}}, \pi_{\boldsymbol{V}}\right)$. We denote by $\operatorname{vec}\left(\Lambda_{\boldsymbol{L}}\right)=\left\{\operatorname{vec}(\Lambda(B)): B \in \mathcal{B}_{b}\left(\mathbb{S}_{d}^{++} \times \mathbb{R}\right)\right\}$ the $\mathbb{R}^{d^{2}}$. valued Lévy basis corresponding to $\Lambda_{L}$ and we rewrite $\left(\Sigma_{t}, t \in \mathbb{R}\right)$ as follows:

$$
\operatorname{vec}\left(\boldsymbol{\Sigma}_{t}\right) \stackrel{d}{=} \int_{\mathbb{S}_{d}^{+}} \int_{\mathbb{R}} \mathbb{I}_{[0, \infty)}(t-s) e^{-(t-s)\left(\boldsymbol{A} \otimes \mathbf{I}_{d \times d}+\mathbf{I}_{d \times d} \otimes \boldsymbol{A}\right)} \operatorname{vec}\left(\Lambda_{\boldsymbol{L}}(d \boldsymbol{A}, d s)\right),
$$

which can also be interpreted as a $d^{2}$-dimensional Lévy-driven MMA process which is mixing (Fuchs and Stelzer 2013, Theorem 3.5).

\section{A.12 Proof of Proposition 6.3.2}

Proof of Proposition 6.3.2 Recall that

$$
\operatorname{vec}\left(\int_{a}^{b} e^{-(b-s) \mathbf{Q}} \boldsymbol{\Sigma}_{s} e^{-(b-s) \mathbf{Q}^{\top}} d s\right)=\int_{a}^{b} e^{-(b-s)\left(\mathbf{Q} \otimes \mathbf{I}_{d \times d}+\mathbf{I}_{d \times d} \otimes \mathbf{Q}\right)} \operatorname{vec}\left(\boldsymbol{\Sigma}_{s}\right) d s .
$$

Using the stationarity of $\left(\boldsymbol{\Sigma}_{t}\right)$ and Eq. (18), by Fubini's theorem we have

$$
\begin{aligned}
\int_{a}^{b} & e^{-(b-s)\left(\mathbf{Q} \otimes \mathbf{I}_{d \times d}+\mathbf{I}_{d \times d} \otimes \mathbf{Q}\right)} \operatorname{vec}\left(\boldsymbol{\Sigma}_{s}\right) d s \\
= & \int_{a}^{b} e^{-(b-s)\left(\mathbf{Q} \otimes \mathbf{I}_{d \times d}+\mathbf{I}_{d \times d} \otimes \mathbf{Q}^{\top}\right)} \int_{-\infty}^{s} e^{-(s-u)\left(\boldsymbol{V} \otimes \mathbf{I}_{d \times d}+\mathbf{I}_{d \times d} \otimes \boldsymbol{V}^{\top}\right)} \operatorname{vec}\left(d \boldsymbol{L}_{u}\right) d s \\
= & \int_{a}^{b} e^{-(b-s)\left(\mathbf{Q} \otimes \mathbf{I}_{d \times d}+\mathbf{I}_{d \times d} \otimes \mathbf{Q}^{\top}\right)} \int_{-\infty}^{0} e^{-v\left(\boldsymbol{V} \otimes \mathbf{I}_{d \times d}+\mathbf{I}_{d \times d} \otimes \boldsymbol{V}^{\top}\right)} \operatorname{vec}\left(d \boldsymbol{L}_{v}\right) d s \\
= & \int_{-\infty}^{0} \int_{a}^{b} e^{-(b-s)\left(\mathbf{Q} \otimes \mathbf{I}_{d \times d}+\mathbf{I}_{d \times d} \otimes \mathbf{Q}^{\top}\right)} e^{-v\left(\boldsymbol{V} \otimes \mathbf{I}_{d \times d}+\mathbf{I}_{d \times d} \otimes \boldsymbol{V}^{\top}\right)} d s \times \operatorname{vec}\left(d \boldsymbol{L}_{v}\right) \\
= & \rho^{-1}(\mathbf{Q})\left[\mathbf{I}_{d \times d}-e^{-(b-a)\left(\mathbf{Q} \otimes \mathbf{I}_{d \times d}+\mathbf{I}_{d \times d} \otimes \mathbf{Q}^{\top}\right)}\right] \int_{-\infty}^{0} e^{-v\left(\boldsymbol{V} \otimes \mathbf{I}_{d \times d}+\mathbf{I}_{d \times d} \otimes \boldsymbol{V}^{\top}\right)} \operatorname{vec}\left(d \boldsymbol{L}_{v}\right) .
\end{aligned}
$$

Using Eqs. (16) and (17), we apply Corollary 4.1, Basse-O'Connor et al. (2014) to have that

$$
\int_{a}^{b} e^{-(b-s) \mathbf{Q}} \boldsymbol{\Sigma}_{s} e^{-(b-s) \mathbf{Q}^{\top}} d s<\infty, \text { a.s. }
$$

for any $a<b \in \mathbb{R} \cup\{ \pm \infty\}$. Indeed, the first and second conditions are true by assumption and definition of the subordinator $\boldsymbol{L}$ whilst the third condition is true if we consider equivalently the truncation functions $\tau(z):=\mathbb{I}_{\left\{\boldsymbol{x} \in \mathbb{R}^{d}:\|\boldsymbol{x}\| \leq 1\right\}}(\boldsymbol{z})$ on $\mathbb{R}^{d}$ and $\tau_{d}(\boldsymbol{Z}):=\mathbb{I}_{\left\{\boldsymbol{X} \in \mathcal{M}_{d}(\mathbb{R}):\|\boldsymbol{X}\| \leq 1\right\}}(\boldsymbol{Z})$ on $\mathcal{M}_{d}(\mathbb{R})$ instead of the truncation function $\tilde{\tau}(\boldsymbol{x}) \equiv 0$ on both $\mathbb{R}^{d}$ and $\mathcal{M}_{d}(\mathbb{R})$ as given in Part 1, Barndorff-Nielsen and Shiryaev (2015) and used in Sect. 6.3.2. By the independence between $\boldsymbol{L}$ and $\mathbb{W}$, we obtain that

$$
\mathrm{E}\left(e^{i \boldsymbol{u}^{\top} \mathbb{F}_{a b}^{(1)}} \mid \sigma\left(\left\{\boldsymbol{\Sigma}_{s}, s \in[a, b]\right\}\right)\right)=\exp \left\{-\frac{1}{2} \boldsymbol{u}^{\top}\left(\int_{a}^{b} e^{-(b-s) \mathbf{Q}} \boldsymbol{\Sigma}_{s} e^{-(b-s) \mathbf{Q}^{\top}} d s\right) \boldsymbol{u}\right\},
$$


where $\sigma\left(\left\{\boldsymbol{\Sigma}_{s}, s \in[a, b]\right\}\right)$ is the $\sigma$-algebra generated by $\left(\boldsymbol{\Sigma}_{s}, a \leq s \leq b\right)$. Hence, for some $c_{1}>0$ small enough, we have for $\|u\| \leq c_{1}$

$$
\mathrm{E}\left(e^{i \boldsymbol{u}^{\top} \mathbb{F}_{a b}^{(1)}} \mid \sigma\left(\left\{\boldsymbol{\Sigma}_{s}, s \in[a, b]\right\}\right)\right) \leq 1-\frac{1}{2} \boldsymbol{u}^{\top}\left(\int_{a}^{b} e^{-(b-s) \mathbf{Q}} \boldsymbol{\Sigma}_{s} e^{-(b-s) \mathbf{Q}^{\top}} d s\right) \boldsymbol{u} \text { a.s. }
$$

Let $c_{2}:=\frac{1}{2} \min _{i, j}\left(\int_{a}^{b} e^{-(b-s) \mathbf{Q}} \mathrm{E}\left(\boldsymbol{\Sigma}_{1}\right) e^{-(b-s) \mathbf{Q}^{\top}} d s\right)_{i j}$ and then, by Fubini's theorem, the distribution of $\mathbb{F}_{a b}^{(1)}$ is non-degenerate since

$$
\mathrm{E}\left(e^{i \boldsymbol{u}^{\top} \mathbb{F}_{a b}^{(1)}}\right) \leq 1-c_{2}\|\boldsymbol{u}\|^{2}, \quad \text { for any }\|\boldsymbol{u}\| \leq c_{1},
$$

for some $c_{2}>0$ according to Proposition 24.19, Sato et al. (1999).

\section{A.13 Proof of Proposition 6.3.3}

Proof of Proposition 6.3.3 Let $k \in \mathbb{N}, 0 \leq t_{1}<\cdots<t_{k}$ and $h>0$. Then

$$
\left(\mathbb{F}_{t_{1}+h}^{(1)}, \ldots, \mathbb{F}_{t_{k}+h}^{(1)}\right) \stackrel{d}{=}\left(\int_{-\infty}^{t_{i}+h} e^{-\left(t_{i}+h-s_{i}\right) \mathbf{Q}} \Sigma_{s_{i}}^{1 / 2} d \mathbb{W}_{s_{i}}, i \in\{1, \ldots, k\}\right),
$$

and defining $u_{i}=s_{i}-\left(t_{i}+h\right)+t_{1}$ for all $i \in\{1, \ldots, k\}$ yields

$$
\left(\mathbb{F}_{t_{1}+h}^{(1)}, \ldots, \mathbb{F}_{t_{k}+h}^{(1)}\right) \stackrel{d}{=}\left(\int_{-\infty}^{t_{1}} e^{-\left(t_{1}-u_{i}\right) \mathbf{Q}} \Sigma_{u_{i}+h+t_{i}-t_{1}}^{1 / 2} d \mathbb{W}_{u_{i}+t_{i}-t_{1}+h}, i \in\{1, \ldots, k\}\right) .
$$

By strict stationarity of $\left(\Sigma_{t}\right)$, we have

$$
\left(\boldsymbol{\Sigma}_{u_{1}+h}, \ldots, \boldsymbol{\Sigma}_{u_{k}+t_{k}-t_{1}+h}\right) \stackrel{d}{=}\left(\boldsymbol{\Sigma}_{u_{1}}, \ldots, \boldsymbol{\Sigma}_{u_{k}+t_{k}-t_{1}}\right),
$$

and we conclude that

$$
\left(\mathbb{F}_{t_{1}+h}^{(1)}, \ldots, \mathbb{F}_{t_{k}+h}^{(1)}\right) \stackrel{d}{=}\left(\mathbb{Y}_{t_{1}}, \ldots, \mathbb{Y}_{t_{k}}\right),
$$

and this result on finite-dimensional distributions gives the strict stationarity of $\mathbb{F}^{(1)}$.

\section{A.14 Proof of Proposition 6.4.2}

Proof of Proposition 6.4.2 We define $\pi_{\mathbf{Q}}:=\delta_{\mathbf{Q}}$, a probability measures on $\mathbb{S}_{d}^{++}$, where $\delta$ is the Dirac delta distribution. According to Sect. 6.2, there exist an $\mathbb{R}^{d}$-valued Lévy basis $\Lambda_{\mathbb{J}}$ on $\mathbb{S}_{d}^{++} \times \mathbb{R}$ associated with the quadruplet $\left(T \times \boldsymbol{\gamma}_{\mathfrak{J}}, \mathbf{0}, T \otimes \nu_{\mathfrak{J}}, \pi_{\mathbf{Q}}\right)$. By independence between $\left(\mathbb{J}_{t}\right)$ and $\left(T_{t}\right)$, we obtain that

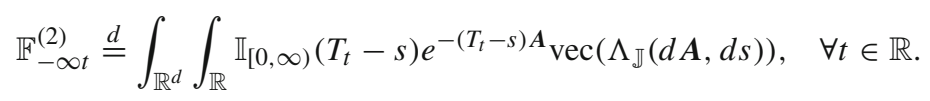

Both of those quantities can be interpreted as Lévy-driven MMA process similarly to Equation (4.5), Fuchs and Stelzer (2013). Therefore, it is mixing by Theorem 3.5, Fuchs and Stelzer (2013). 


\section{A.15 Proof of Proposition 6.5.2}

Proof of Proposition 6.5.2 Using Proposition 6.3.1 and the proof of Proposition 6.3.2, recall that $\left(\Sigma_{t}, t \in \mathbb{R}\right)$ is an MMA process hence mixing (Fuchs and Stelzer 2013, Theorem 2.5) Theorem such that for all $t \in \mathbb{R} \cup\{\infty\}$

$$
\int_{-\infty}^{t} e^{-(t-s) \mathbf{Q}} \Sigma_{s} e^{-(t-s) \mathbf{Q}^{\top}} d s<\infty \quad \text { a.s. }
$$

as a Lebesgue integral of $\left(\boldsymbol{\Sigma}_{t}\right) \omega$-wise (see Eq. (18)). Also, recall that $\left(\mathbb{F}_{-\infty t}^{(1)}, t \in \mathbb{R}\right)$ is stationary by Proposition 6.3.3. From the integrability of $\left(\Sigma_{t}\right)$, we have that $\left(\mathbb{F}_{-\infty t}^{(1)}, t \in \mathbb{R}\right)$ is a centred Gaussian process where its autocovariance function $\operatorname{Acov}(h):=\mathrm{E}\left(\mathbb{F}_{-\infty t}^{(1)} \mathbb{F}_{-\infty t+h}^{(1)}\right)$ for $h \geq 0$ (and some $t \in \mathbb{R}$ ) is given by

$$
\begin{aligned}
& \operatorname{Acov}(h)=\mathrm{E}\left[\int_{-\infty}^{t} e^{-(t-u) \mathbf{Q}} \Sigma_{u}^{1 / 2} d \mathbb{W}_{u}\left(\int_{-\infty}^{t+h} e^{-(t+h-v) \mathbf{Q}} \Sigma_{v}^{1 / 2} d \mathbb{W}_{v}\right)^{\top}\right] \\
& =\left[\int_{-\infty}^{t} e^{-(t-u) \mathbf{Q}} \mathrm{E}\left(\boldsymbol{\Sigma}_{u}\right) e^{-(t-u) \mathbf{Q}^{\top}}\right] e^{-h \mathbf{Q}^{\top}}, \quad \text { by Fubini's theorem, } \\
& =O\left(e^{-h \mathbf{Q}^{\top}}\right)=o(1), \quad \text { as } h \rightarrow \infty \text { since } \mathrm{E}\left(\boldsymbol{\Sigma}_{t}\right)=-\rho^{-1}\left(\mathrm{E}\left(\boldsymbol{L}_{1}\right)\right)<\infty \text {, }
\end{aligned}
$$

where we recall that $\rho(\boldsymbol{X})=\boldsymbol{V} \boldsymbol{X}+\boldsymbol{X} \boldsymbol{V}^{\top}$.

According to Sections 3.7 and 4.12, Dym and McKean (2008), given that $\mathbb{F}^{(1)}$ is stationary and a centred Gaussian process such that its autocovariance Acov is continuous and such that $\operatorname{Acov}(h)=o(1)$ as $h \rightarrow \infty$, we conclude that $\left(\mathbb{F}_{-\infty t}^{(1)}\right)$ is mixing. Therefore, by Proposition 6.4.2, $\left(\mathbb{Y}_{t}, t \in \mathbb{R}\right)$ is the sum of two mixing processes hence itself mixing and ergodic.

\section{References}

Barndorff-Nielsen OE, Pérez-Abreu V (2008) Matrix subordinators and related Upsilon transformations. Theory Probab Its Appl 52(1):1-23

Barndorff-Nielsen OE, Shephard N (2001) Non-Gaussian Ornstein-Uhlenbeck-based models and some of their uses in financial economics. J R Stat Soc Ser B (Stat Methodol) 63(2):167-241

Barndorff-Nielsen OE, Stelzer R (2007) Positive-definite matrix processes of finite variation. Probab Math Stat 27(1):3-43

Barndorff-Nielsen OE, Stelzer R (2011) Multivariate supOU processes. Ann Appl Probab 21(1):140-182

Barndorff-Nielsen OE, Shiryaev A (2015) Change of time given by subordinators. In: Change of time and change of measure, vol 21. World Scientific Publishing Company, pp 158-162

Barndorff-Nielsen OE, Veraart AED (2012) Stochastic volatility of volatility and variance risk premia. J Financ Econom 11(1):1-46. https://doi.org/10.1093/jjfinec/nbs008

Barndorff-Nielsen OE, Benth FE, Veraart AED (2018) Ambit Stochastics, vol 5. Springer, Berlin, pp 153-202

Basak GK, Lee P et al (2008) Asymptotic properties of an estimator of the drift coefficients of multidimensional Ornstein-Uhlenbeck processes that are not necessarily stable. Electron J Stat 2:1309-1344

Basse-O'Connor A, Graversen S-E, Pedersen J (2014) Stochastic integration on the real line. Theory Probab Appl 58(2):193-215

Belomestny D, Trabs M, Tsybakov AB (2019) Sparse covariance matrix estimation in high-dimensional deconvolution. Bernoulli 25(3):1901-1938

Boninsegna L, Nüske F, Clementi C (2018) Sparse learning of stochastic dynamical equations. J Chem Phys 148(24):241723. https://doi.org/10.1063/1.5018409

Brockwell PJ (2009) Lévy-Driven Continuous-time ARMA processes. Springer, Berlin, pp 457-480. https:// doi.org/10.1007/978-3-540-71297-8_20 
Brockwell PJ, Davis RA, Yang Y (2007) Estimation for nonnegative Lévy-driven Ornstein-Uhlenbeck processes. J Appl Probab 44(4):977-989

Brownlees C, Nualart E, Sun Y (2020) On the estimation of integrated volatility in the presence of jumps and microstructure noise. Economet Rev 39(10):991-1013

Bühlmann P, Van De Geer S (2011) Statistics for high-dimensional data: methods, theory and applications. Springer, Cham

Cai TT, Ren Z, Zhou HH (2016) Estimating structured high-dimensional covariance and precision matrices: optimal rates and adaptive estimation. Electron J Stat 10(1):1-59

Chen CY-H, Härdle WK, Okhrin Y (2019) Tail event driven networks of SIFIs. J Econom 208(1):282-298 (Special Issue on Financial Engineering and Risk Management)

Chen EY, Fan J, Zhu X (2020) Community network auto-regression for high-dimensional time series

Cont R, Tankov P (2004) Stochastic volatility models with jumps. In: Financial modelling with jump processes. Chapman \& Hall pp 496-497

Courgeau V, Veraart AED (2020) High-frequency estimation of the lévy-driven graph ornstein-uhlenbeck process

Culver WJ (1966) On the existence and uniqueness of the real logarithm of a matrix. Proc Am Math Soc 17(5):1146-1151

Dereich S, Mörters P (2013) Random networks with sublinear preferential attachment: the giant component. Ann Probab 41(1):329-384

Dym H, McKean H (2008) Gaussian processes, function theory, and the inverse spectral problem. Dover books on mathematics. Dover Publications, London

Endres S, Stübinger J (2019) Optimal trading strategies for Lévy-driven Ornstein-Uhlenbeck processes. Appl Econ 51(29):3153-3169. https://doi.org/10.1080/00036846.2019.1566688

Fasen V (2013) Statistical estimation of multivariate Ornstein-Uhlenbeck processes and applications to cointegration. J Econom 172(2):325-337

Fuchs F, Stelzer R (2013) Mixing conditions for multivariate infinitely divisible processes with an application to mixed moving averages and the supOU stochastic volatility model. ESAIM Probab Stat 17:455-471

Gaïffas S, Matulewicz G (2019) Sparse inference of the drift of a high-dimensional Ornstein-Uhlenbeck process. J Multivariate Anal 169:1-20

Gushchin A, Pavlyukevich I, Ritsch M (2020) Drift estimation for a lévy-driven ornstein-uhlenbeck process with heavy tails. Stat Infer Stoch Process 23(3):553-570. https://doi.org/10.1007/s11203-020-09210-8

Hájek J (1970) A characterization of limiting distributions of regular estimates. Zeitschrift für Wahrscheinlichkeitstheorie und Verwandte Gebiete 14(4):323-330. https://doi.org/10.1007/BF00533669

Holý V, Tomanová P (2018) Estimation of Ornstein-Uhlenbeck process using ultra-high-frequency data with application to intraday pairs trading strategy. arXiv preprint arXiv:1811.09312

Höpfner R (2014) Some stochastic process examples for local asymptotics of type LAN, LAMN and LAQ. In: Asymptotic statistics. De Gruyter, Berlin, pp 212-242

Jacod J (1979) Changements de temps et changements d'espace. In: Calcul Stochastique et Problémes de Martingales. Springer Berlin, pp 311-336. https://doi.org/10.1007/BFb0064918

Kallsen J, Shiraev A (2002) Time change representation of stochastic integrals. Theory Probab Appl 46(3):522528

Kevei P (2018) Ergodic properties of generalized Ornstein-Uhlenbeck processes. Stoch Process Appl 128(1):156-181

Kim D, Wang Y, Zou J (2016) Asymptotic theory for large volatility matrix estimation based on high-frequency financial data. Stoch Process Appl 126(11):3527-3577

Knight M, Leeming K, Nason G, Nunes M (2020) Generalized network autoregressive processes and the GNAR package. J Stat Softw 96(5):1-36

Knight M, Nunes M, Nason G (2016) Modelling, detrending and decorrelation of network time series. arXiv preprint arXiv:1603.03221

Küchler U, Sørensen M (1997) Exponential families of stochastic processes. Springer, New York

Le Cam L, Lo Yang G (1990) Locally asymptotically normal families. Springer, New York, pp 52-98

Longoria G, Davy A, Shi L (2018) Ornstein-Uhlenbeck-Lévy electricity portfolios with wind energy contracting. Technol Econ Smart Grids Sustain Energy 3(1). Article number 16. https://doi.org/10.1007/s40866018-0054-9

Ma Y, Guo S, Wang H (2021) Sparse spatio-temporal autoregressions by profiling and bagging. J Econom (to appear)

Mai H (2014) Efficient maximum likelihood estimation for Lévy-driven Ornstein-Uhlenbeck processes. Bernoulli 20(2):919-957

Mancini C (2009) Non-parametric threshold estimation for models with stochastic diffusion coefficient and jumps. Scand J Stat 36(2):270-296 
Maruyama G (1970) Infinitely divisible processes. Theory Probab Appl 15(1):1-22

Masuda H (2004) On multidimensional Ornstein-Uhlenbeck processes driven by a general Lévy process. Bernoulli 10(1):97-120

Masuda H (2007) Ergodicity and exponential $\beta$-mixing bounds for multidimensional diffusions with jumps. Stoch Process Appl 117(1):35-56

Masuda H (2010) Approximate self-weighted LAD estimation of discretely observed ergodic OrnsteinUhlenbeck processes. Electron J Stat 4:525-565. https://doi.org/10.1214/10-EJS565

Melanson A, Longtin A (2019) Data-driven inference for stationary jump-diffusion processes with application to membrane voltage fluctuations in pyramidal neurons. J Math Neurosci 9(1). Article number 6. https:// doi.org/10.1186/s13408-019-0074-3

Morales D, Pardo L, Vajda I (2000) Rényi statistics in directed families of exponential experiments. Stat J Theor Appl Stat 34(2):151-174

Pap G, van Zuijlen MC (1996) Parameter estimation with exact distribution for multidimensional OrnsteinUhlenbeck processes. J Multivar Anal 59(2):153-165

Pigorsch C, Stelzer R (2009a) A multivariate Ornstein-Uhlenbeck type stochastic volatility model (preprint)

Pigorsch C, Stelzer R (2009b) On the definition, stationary distribution and second order structure of positive semidefinite Ornstein-Uhlenbeck type processes. Bernoulli 15(3):754-773. https://doi.org/10.3150/08BEJ175

Rajput BS, Rosinski J (1989) Spectral representations of infinitely divisible processes. Probab Theory Relat Fields 82(3):451-487. https://doi.org/10.1007/BF00339998

Rosiński J, Żak T (1997) The equivalence of ergodicity and weak mixing for infinitely divisible processes. J Theor Probab 10(1):73-86

Sandrić N (2016) Ergodicity of Lévy-Type processes. ESAIM: PS 20:154-177. https://doi.org/10.1051/ps/ 2016009

Sato K-I, Yamazato M (1984) Operator-selfdecomposable distributions as limit distributions of processes of Ornstein-Uhlenbeck type. Stoch Process Appl 17(1):73-100

Sato K-I, Ken-Iti S, Katok A (1999) Lévy processes and infinitely divisible distributions. Cambridge University Press, Cambridge

Sims CA (1980) Macroeconomics and reality. Econometrica 48(1):1-48

Sørensen M (1991) Likelihood methods for diffusions with jumps. Stat Inference Stoch Process, pp 67-105

Tao M, Wang Y, Zhou HH (2013) Optimal sparse volatility matrix estimation for high-dimensional Itô processes with measurement errors. Ann Stat 41(4):1816-1864

Yamazato M (1983) Absolute continuity of operator-self-decomposable distributions on $\mathbb{R}^{d}$. J Multivariate Anal 13(4):550-560

Yang X, Wu Y, Zheng Z, Hu J-Q (2020) Method of Moments Estimation For Lévy-driven Ornstein-Uhlenck Stochastic Volatility Models. Probab Eng Inform Sci, 1-30

Zhu X, Pan R, Li G, Liu Y, Wang H et al (2017) Network vector autoregression. Ann Stat 45(3):1096-1123

Zhu X, Huang D, Pan R, Wang H (2020) Multivariate spatial autoregressive model for large scale social networks. J Econom 215(2):591-606

Zou H (2006) The adaptive lasso and its oracle properties. J Am Stat Assoc 101(476):1418-1429

Publisher's Note Springer Nature remains neutral with regard to jurisdictional claims in published maps and institutional affiliations. 